\title{
The Alterations and Potential Roles of MCMs in Breast Cancer
}

\author{
Xinyu Liu $\mathbb{D},{ }^{1}$ Ying Liu, ${ }^{1,2}$ Qiangshan Wang, ${ }^{3}$ Siqi Song, ${ }^{1}$ Lingjun Feng $\mathbb{D}^{4}{ }^{4}$ \\ and Chunying Shi $\mathbb{B}^{1}$ \\ ${ }^{1}$ School of Basic Medicine, College of Medicine, Qingdao University, Qingdao 266071, China \\ ${ }^{2}$ Institute for Translational Medicine, The Affiliated Hospital of Qingdao University, College of Medicine, Qingdao University, \\ Qingdao 266021, China \\ ${ }^{3}$ Jiaozhou Maternal and Child Health Hospital, Qingdao 266300, China \\ ${ }^{4}$ Thyroid \& Breast Surgery, Hospital of Weifang Medical University, Weifang 261031, China
}

Correspondence should be addressed to Lingjun Feng; wfyxyfsyyflj@163.com and Chunying Shi; cyshi@qdu.edu.cn

Received 13 April 2021; Revised 7 July 2021; Accepted 4 August 2021; Published 24 August 2021

Academic Editor: Nicola Silvestris

Copyright (c) 2021 Xinyu Liu et al. This is an open access article distributed under the Creative Commons Attribution License, which permits unrestricted use, distribution, and reproduction in any medium, provided the original work is properly cited.

\begin{abstract}
The minichromosome maintenance (MCM) protein family plays a key role in eukaryotic DNA replication and has been confirmed to be associated with the occurrence and progression of many tumors. However, the expression levels, functions, and prognostic values of MCMs in breast cancer (BC) have not been clearly and systematically explained. In this article, we studied the transcriptional levels of MCMs in BC based on the Oncomine database. Kaplan-Meier plotter was used to analyze prognostic value of MCMs in human BC patients. Furthermore, we constructed a MCM coexpression gene network and performed functional annotation analysis through DAVID to reveal the functions of MCMs and coexpressed genes. The data showed that the expression of MCM2-8 and MCM10 but not MCM1 and MCM9 was upregulated in BC. Kaplan-Meier plotter analysis revealed that high transcriptional levels of MCM2, MCM4-7, and MCM10 were significantly related to low relapse-free survival (RFS) in BC patients. In contrast, high levels of MCM1 and MCM9 predicted high RFS for BC patients. This study suggests that MCM2, MCM4-7, and MCM10 possess great potential to be valuable prognostic biomarkers for $\mathrm{BC}$ and that MCM1 and MCM9 may serve as potential treatment targets for BC patients.
\end{abstract}

\section{Introduction}

Surveys show that breast cancer $(\mathrm{BC})$ patients diagnosed worldwide are increasing, and $\mathrm{BC}$ is the most common carcinoma type in the female population $[1,2]$. BC can be further divided into four subtypes, including luminal A, luminal B, basal-like, and human epidermal growth factor receptor-2 (HER2) overexpression [3]. Classic clinical prognostic markers, such as progesterone receptor (PR), HER2, and estrogen receptor (ER) have played positive roles in endocrine therapy or targeted therapy in BC patients [4]. Because of the heterogeneity of various tumors, the limitations of the current markers are sensitivity and specificity. Therefore, valuable biomarkers are needed as prognostic predictors to effectively upregulate prognosis and precisely individualized therapy effects.

To date, the roles of minichromosome maintenance (MCM) protein family members identified in human cancers have been widely reported. The MCM family plays important roles in the cell cycle and genome replication, including ten members: serum response factor (SRF, also called MCM1) and MCM2-10 [5, 6]. The MCM2-7 complexes are involved in the formation of the prereplication complex and have helicase activity, which makes the DNA detach and leads to the recruitment of DNA polymerase and the activation of DNA replication [7, 8]. MCMs are also involved in the response of DNA damage $[9,10]$. In addition, MCM interacts with cellular tumor antigen p53 binding protein 1 (53BP1) and Rad51, and the consumption of MCM leads to a reduction in 53BP1 and Rad51 foci formed after DNA damage $[10,11]$. At present, the overexpression of MCM has been detected in various cancer tissues and cancer cell lines, including squamous cell lung carcinoma [12], kidney cancer [13], prostate carcinoma [14], BC [15], digestive system tumors [16-18], brain tumors [19], and lymphomas [20]. 
The abnormal expression of MCMs and its relationship with clinicopathological characteristics and prognosis have been partially reported in human BC. However, bioinformatics analysis has not been performed to systematically explore the role of MCMs in BC. Based on online databases, we analyzed the expression patterns, clinicopathological characteristics, functions, and different prognostic values of MCMs in patients with BC. In addition, potential regulatory miRNA-regulating MCMs were screened, contributing to regulating the expression of MCMs in BC and identifying targets of precise treatment for $\mathrm{BC}$ patients. Our research helps to strengthen and acknowledge of the roles of the MCMs in BC.

\section{Materials and Methods}

2.1. Oncomine Analysis. Oncomine [21, 22] (https://www. oncomine.org/resource/main.html) provides gene data that can be used to reveal the expression of target genes in various cancers. The mRNA expression level of MCMs in cancer samples was compared with normal samples. The threshold of $p$ value is 0.05 , fold change is 2 , and gene rank is top $5 \%$.

2.2. GEPIA Analysis. GEPIA [23] (http://gepia.cancer-pku. $\mathrm{cn} /$ ) can be used to analyze the RNA expression of various cancer and normal tissue samples based on TCGA and GTEx. GEPIA was used to perform the correlation analysis of MCMs in BC.

2.3. Survival Analysis. Kaplan-Meier plotter [24] (http:// www.kmplot.com/) can be used to predict the impact of target genes on the survival rate of patients with different cancer types. We use it to analyze the prognostic value of MCMs and their regulatory miRNAs in BC.

2.4. cBioPortal Analysis. The Invasive Breast Carcinoma database (METABRIC, Nature 2012 and Nat Commun 2016, including 2509 samples) was selected to analyze and construct the cancer genome atlas of MCMs based on cBioPortal [25] (https://www.cbioportal.org). Mutations, putative copy-number alterations from DNA copy, and mRNA expression (microarray) $\mathrm{z}$-scores relative to diploid samples of the genomic profiles were chosen to be analyzed.

2.5. STRING Analysis. We used STRING [26] database (https://string-db.org) to establish a protein-protein network that showed the coexpression relationships between MCMs and other nodes.

2.6. Function Annotation Analysis. DAVID [27] (https:// david.ncifcrf.gov/) was used to analyze the MCMs and coexpressed genes to identify GO terms and to visualize genes on Kyoto Encyclopedia of Genes and Genomes (KEGG) pathway maps. $p<0.05$ was considered to be statistically significant.
2.7. ENCORI Analysis. ENCORI [28] (http://starbase.sysu. edu.cn/panCancer.php) was used to predict miRNAs that regulated MCMs and determine the expression levels of miRNA-regulating MCMs. The parameters used in this research were medium stringency $(\geq 3)$ and 1 cancer type.

2.8. Statistical Analysis. The mRNA expression level of MCMs between BC and normal samples was detected to reveal the statistical difference by Student's $t$-tests. Survival curves of various subtypes in BC patients with different expression level of MCMs were drafted based on log-rank test and hazard ratio (HR) by Kaplan-Meier plotter. $p<0.05$ was considered to be statistically significant.

\section{Results}

3.1. The Transcriptional Levels of MCMs and Clinicopathological Characteristics in BC Patients. Alterations in the transcriptional levels of MCM family members, including MCM1-10, have been widely reported in cancers. Oncomine data showed that the mRNA expression of MCM2-8 and MCM10 but not MCM1 and MCM9 in BC samples was significantly upregulated compared with normal samples (Figures 1 and 2). As shown in Table 1, previous reports indicated that MCM2-8 and MCM10 were upregulated in BC [3, 29-35]. The expression levels of MCMs with tumor stage for breast cancer were analyzed by Gene Expression Profiling Interactive Analysis (GEPIA) database. As shown in Figure 3, MCM2, MCM3, MCM7, and MCM10 groups significantly varied, whereas MCM1, MCM4, MCM5, MCM6, MCM8, and MCM9 groups did not significantly differ.

\subsection{Diagnostic and Prognostic Value of MCMs in Clinical BC} Patients. Using Kaplan-Meier plotter, we analyzed the prognostic value of MCMs in BC patients and drew related survival maps (Figure 4). Elevated mRNA expression levels of MCM2, MCM4-7, and MCM10 were significantly related to short relapse-free survival (RFS), whereas MCM3 and MCM8 did not, indicating that they were related to poor prognosis in BC patients. However, the decreased expression of MCM1 and MCM9 in BC was significantly related to prolonged RFS, indicating that they were related to good prognosis in $\mathrm{BC}$ patients.

3.3. Alterations, Gene Correlations, and Coexpression Gene Network of MCMs in BC. Using the cBioPortal database, we analyzed alterations of MCMs in BC. As shown in Figure 5(a), MCMs were altered in 836 of the 2509 BC samples (33\%). The GEPIA database was used to analyze the correlations of the mRNA expressions of MCMs in BC. As shown in Figure 5(b), there were significant positive correlations $(R>0.3)$ between following MCMs: MCM2 with MCM3-8 and MCM10; MCM3 with MCM4-8 and MCM10; MCM4 with MCM5-8 and MCM10; MCM5 with MCM6-8 and MCM10; MCM6 with MCM7, MCM8, and 


\begin{tabular}{|l|}
\hline \\
Analysis Type by Cancer \\
\hline Bladder Cancer \\
\hline Brain and CNS Cancer \\
\hline Breast Cancer \\
\hline Cervical Cancer \\
\hline Colorectal Cancer \\
\hline Esophageal Cancer \\
\hline Gastric Cancer \\
\hline Head and Neck Cancer \\
\hline Kidney Cancer \\
\hline Leukemia \\
\hline Liver Cancer \\
\hline Lung Cancer \\
\hline Lymphoma \\
\hline Melanoma \\
\hline Myeloma \\
\hline Other Cancer \\
\hline Ovarian Cancer \\
\hline Pancreatic Cancer \\
\hline Prostate Cancer \\
\hline Sarcoma \\
\hline Significant Unique Analyses \\
\hline Total Unique Analyses \\
\hline
\end{tabular}
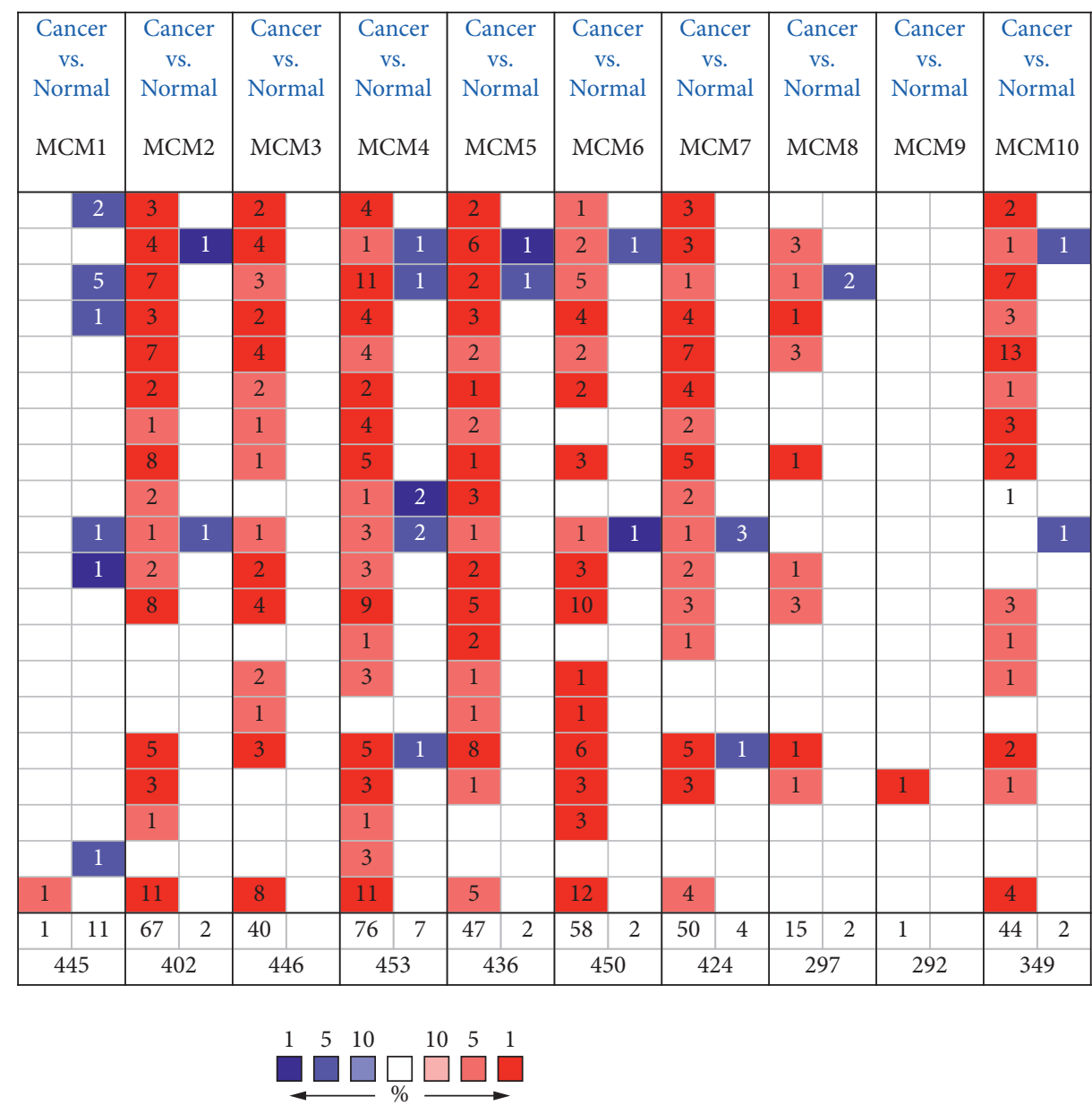

FIgURE 1: The transcriptional levels of MCMs in various cancers. The figure shows the number of datasets with higher expression levels of MCMs in various types of carcinoma samples compared to normal samples. Red cells represent high mRNA transcriptional level and blue cells represent low mRNA transcriptional level. The threshold of $p$ value is 0.05 , fold change is 2 , and gene rank is top $5 \%$.

MCM10; MCM7 with MCM8 and MCM10; and MCM8 with MCM9 and MCM10.

Furthermore, we constructed a coexpressed gene network of MCMs (Figure 6(a)). In addition, the potential functions of MCMs and coexpressed genes significantly related to MCMs were predicted by performing Gene Ontology (GO) analysis, including biological processes (BPs), cellular components (CCs), and molecular functions (MFs), and KEGG analysis based on DAVID. We found that GO: 0006260 (DNA replication), GO:0000082 (G1/S transition of mitotic cell cycle), and GO:0006270 (DNA replication initiation) were significantly regulated by alterations in MCMs (Figure 6(b)). Moreover, these MCM alterations significantly affected GO:0005654 (nucleoplasm), GO:0000784 (nuclear chromosome, telomeric region), GO:0005634 (nucleus), GO: 0005664 (nuclear origin of replication recognition complex), GO:0042555 (MCM complex), GO:0000808 (origin recognition complex), and GO:0005658 (alpha DNA polymerase: primase complex) (Figure 6(c)). Alterations in MCMs also significantly controlled GO:0003688 (DNA replication origin binding), GO:0003677 (DNA binding), GO:0003697 (singlestranded DNA binding), GO:0005524 (ATP binding), GO: 0003887 (DNA-directed DNA polymerase activity), GO:
0003682 (chromatin binding), GO:0005515 (protein binding), and GO:0003678 (DNA helicase activity) (Figure 6(d)). The important roles of MCMs in DNA replication and cell cycle have been widely recognized.

KEGG analysis was used to define pathways related to the altered functions of MCMs and frequently altered coexpressed genes. Through KEGG analysis, pathways related to the altered functions of MCMs in BC were discovered (Figure 6(e)). Among these pathways, DNA replication, cell cycle, and P53 signaling pathways were significantly related to the occurrence and progression of BC (Figures 7-9).

3.4. Regulatory miRNAs and Survival Analysis. As shown in Tables S1-S8, 137 miRNAs regulating MCM1, 62 miRNAs regulating MCM2, 62 miRNAs regulating MCM4, 41 miRNAs regulating MCM5, 47 miRNAs regulating MCM6, 40 miRNAs regulating MCM7, 9 miRNAs regulating MCM9, and 63 miRNAs regulating MCM10 were predicted with the ENCORI platform. Among them, 45 miRNA-MCM1 pairs, 22 miRNAMCM2 pairs, 15 miRNA-MCM4 pairs, 15 miRNA-MCM5 pairs, 20 miRNA-MCM6 pairs, 9 miRNA-MCM7 pairs, 6 miRNA-MCM9 pairs, and 23 miRNA-MCM10 pairs were 

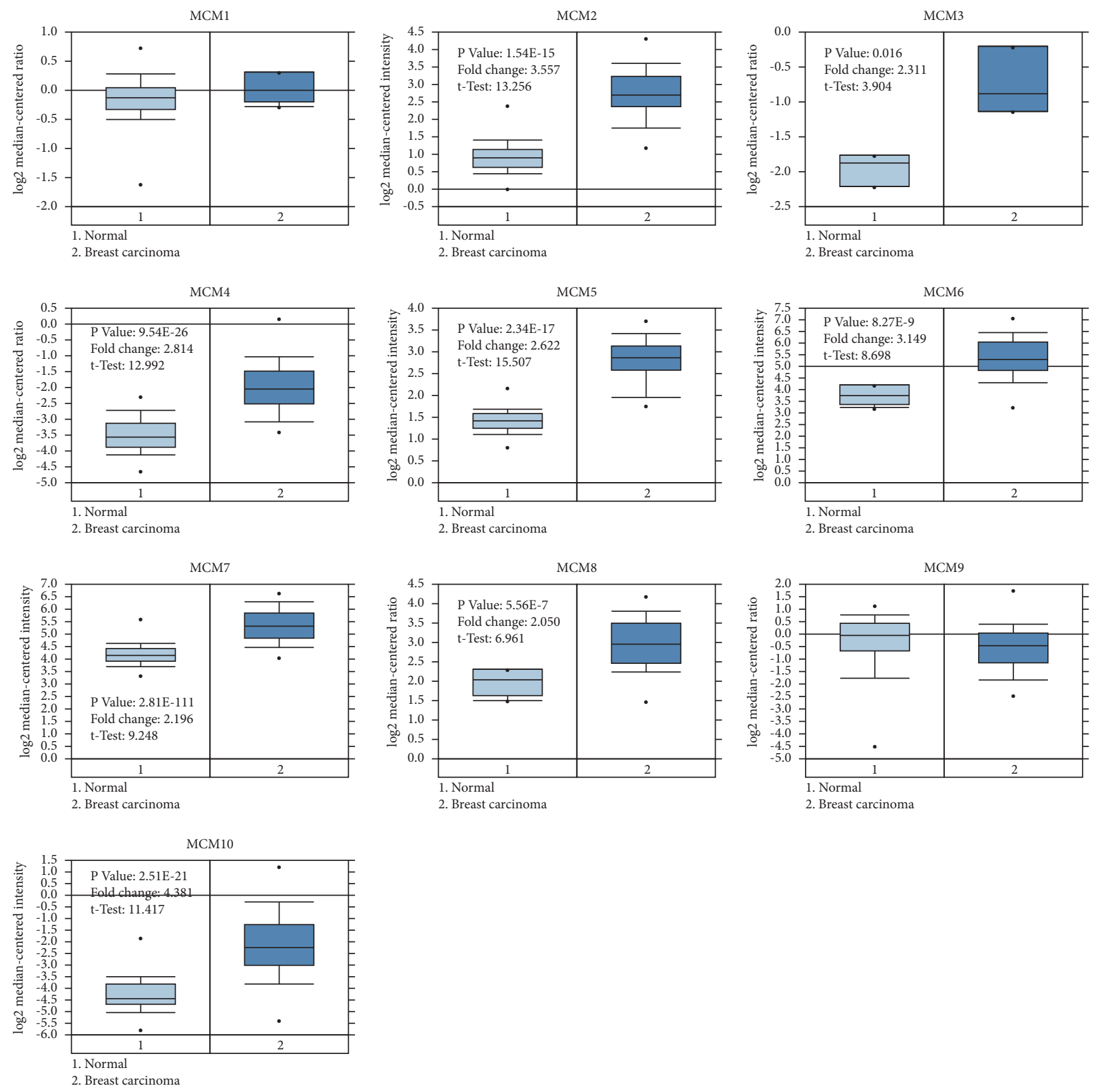

Figure 2: The transcriptional levels of MCMs in BC compared to normal samples based on the Oncomine database.

negatively correlated. Furthermore, the ability to predict poor prognosis in BC patients with MCM1 expression was significant for three miRNAs: hsa-miR-760 ( $p$ value $=0.000825)$, hsa-miR$1224-5 \mathrm{p} \quad(p \quad$ value $=0.0135)$, and hsa-miR-4739 $(p$ val$\mathrm{ue}=0.0368)$ (Figure 10(a)). 10 miRNAs were low expression in $\mathrm{BC}$ tissues, and their low expression predicted good prognosis in $\mathrm{BC}$ patients. These miRNAs negatively regulated MCM2, MCM4, MCM5, and MCM10 (Figures 10(b)-10(e)). Negative miRNA-MCM pairs were as follows: hsa-miR-139-5p-MCM2 $(p$ value $=2.73 E-12)$, hsa-miR-299-3p-MCM4 $(p$ value $=$ $4.64 E-06)$, hsa-miR-654-5p-MCM4 ( $p$ value $=1.28 E-07)$, hsa-miR-140-3p-MCM4 ( $p$ value $=1.48 E-02)$, hsa-miR$139-5 \mathrm{p}-\mathrm{MCM} 5(p$ value $=7.77 E-03)$, hsa-miR-326-MCM5 $(p \quad$ value $=9.16 E-03)$, hsa-miR-654-5p-MCM5 $(p$ value $=$ $1.18 E-06)$, hsa-miR-299-3p-MCM10 ( $p$ value $=7.45 E$
- 04), hsa-miR-485-3p-MCM10 ( $p$ value $=4.55 E-02)$, and hsa-miR-543-MCM10 ( $p$ value $=1.29 E-02)$. The above results suggest that the established miRNA-MCM regulatory networks may be valuable prognostic markers and therapeutic targets for BC (Figures 11(a)-11(e)).

\section{Discussion}

An imbalance in MCM mRNA expression levels has been reported in many types of cancer [12-20]. Although the roles of MCMs in the occurrence, distant metastasis, and prognosis of patients with various tumors have been partially confirmed, the comprehensive biological information of MCMs in BC has not been systematically clarified. This study analyzed the expression levels, clinicopathological 
TABLE 1: The significant changes of MCM expression in transcription level between different types of breast cancer and normal breast tissues (Oncomine database).

\begin{tabular}{|c|c|c|c|c|c|}
\hline & $\begin{array}{c}\text { Type of breast cancer versus normal breast tissue reference invasive } \\
\text { breast carcinoma }\end{array}$ & $\begin{array}{l}\text { Fold } \\
\text { change }\end{array}$ & $p$ value & $T$-test & $\begin{array}{l}\text { Source and/or } \\
\text { reference }\end{array}$ \\
\hline \multirow{4}{*}{ MCM1 } & NA & NA & NA & NA & NA \\
\hline & Medullary breast carcinoma & 3.557 & $1.54 E-15$ & 13.256 & Curtis breast [29] \\
\hline & Invasive ductal breast carcinoma & 2.503 & $3.82 E-92$ & 34.511 & Curtis breast [29] \\
\hline & Breast carcinoma & 2.062 & $4.52 E-6$ & 6.693 & Curtis breast [29] \\
\hline \multirow{5}{*}{ MCM2 } & Mixed lobular and ductal breast carcinoma & 2.200 & $1.18 E-6$ & 8.165 & TCGA \\
\hline & Invasive lobular breast carcinoma & 2.120 & $1.06 E-12$ & 8.432 & TCGA \\
\hline & Invasive ductal breast carcinoma & 2.877 & $5.24 E-31$ & 17.777 & TCGA \\
\hline & Invasive breast carcinoma & 2.184 & $1.30 E-17$ & 9.743 & TCGA \\
\hline & Fibroadenoma & 2.311 & 0.016 & 3.904 & Sorlie breast [3] \\
\hline \multirow{7}{*}{ MCM3 } & Fibroadenoma & 2.535 & 0.019 & 4.415 & Sorlie breast $2[30]$ \\
\hline & Medullary breast carcinoma & 2.101 & $1.62 E-11$ & 9.579 & Curtis breast [21] \\
\hline & Ductal breast carcinoma & 3.081 & $3.36 E-14$ & 13.705 & Perou breast [31] \\
\hline & Lobular breast carcinoma & 2.698 & 0.013 & 4.040 & Perou breast [31] \\
\hline & Invasive ductal breast carcinoma & 3.192 & $3.89 E-48$ & 22.602 & TCGA \\
\hline & Invasive breast carcinoma & 2.814 & $9.54 E-26$ & 12.992 & TCGA \\
\hline & Invasive ductal and lobular carcinoma & 2.990 & $2.27 E-7$ & 13.999 & TCGA \\
\hline \multirow{6}{*}{ MCM4 } & Invasive lobular breast carcinoma & 2.048 & $1.02 E-13$ & 9.018 & TCGA \\
\hline & Medullary breast carcinoma & 3.126 & $1.96 E-13$ & 11.226 & Curtis breast [29] \\
\hline & Invasive ductal breast carcinoma & 2.412 & $1.58 E-85$ & 32.416 & Curtis breast [29] \\
\hline & Invasive breast carcinoma & 2.011 & $4.18 E-6$ & 5.726 & Curtis breast [29] \\
\hline & Invasive breast carcinoma & 2.199 & $5.37 E-6$ & 9.490 & Gluck breast [32] \\
\hline & Ductal breast carcinoma & 3.806 & $7.88 E-8$ & 8.590 & $\begin{array}{c}\text { Richardson breast } 2 \\
{[33]}\end{array}$ \\
\hline \multirow{4}{*}{ MCM5 } & Medullary breast carcinoma & 2.622 & $2.34 E-17$ & 15.507 & Curtis breast [29] \\
\hline & Intraductal cribriform breast adenocarcinoma & 2.082 & $2.53 E-4$ & 5.919 & TCGA \\
\hline & Ductal breast carcinoma & 3.149 & $8.27 E-9$ & 8.698 & $\begin{array}{c}\text { Richardson breast } 2 \\
{[33]}\end{array}$ \\
\hline & Medullary breast carcinoma & 2.551 & $1.26 E-12$ & 10.679 & Curtis breast [21] \\
\hline \multirow{3}{*}{ MCM6 } & Invasive lobular breast carcinoma & 3.692 & 0.009 & 2.828 & Radvanyi breast [34] \\
\hline & Invasive ductal breast carcinoma & 3.329 & 0.012 & 2.737 & Radvanyi breast [34] \\
\hline & Invasive ductal breast carcinoma & 2.358 & 0.007 & 3.143 & Turashvili breast [35] \\
\hline MCM7 & Medullary breast carcinoma & 2.196 & $2.81 E-12$ & 9.248 & Curtis breast [29] \\
\hline MCM8 & Ductal breast carcinoma & 2.050 & $5.56 E-9$ & 6.961 & $\begin{array}{c}\text { Richardson breast } 2 \\
{[33]}\end{array}$ \\
\hline \multirow{4}{*}{ MCM9 } & NA & NA & NA & NA & NA \\
\hline & Male breast carcinoma & 4.716 & $5.49 E-26$ & 20.205 & TCGA \\
\hline & Invasive ductal breast carcinoma & 4.774 & $1.21 E-45$ & 21.470 & TCGA \\
\hline & Invasive breast carcinoma & 4.381 & $2.51 E-21$ & 11.417 & TCGA \\
\hline \multirow{4}{*}{ MCM10 } & Invasive lobular breast carcinoma & 2.510 & $4.92 E-11$ & 7.582 & TCGA \\
\hline & Ductal breast carcinoma & 7.508 & $1.68 E-12$ & 9.492 & $\begin{array}{c}\text { Richardson breast } 2 \\
{[33]}\end{array}$ \\
\hline & Invasive ductal breast carcinoma & 2.077 & $2.99 E-92$ & 29.777 & Curtis breast [29] \\
\hline & Medullary breast carcinoma & 3.802 & $3.90 E-13$ & 11.272 & Curtis breast [29] \\
\hline
\end{tabular}

NA, not available; TCGA, the Cancer Genome Atlas.

characteristics, functions, and prognostic values of MCMs in $\mathrm{BC}$ to aid in the treatment design of $\mathrm{BC}$ patients and prognostic accuracy.

The MCM2-8 and MCM10 expression levels were upregulated in $\mathrm{BC}$, but high expression of MCM1 and MCM9 in BC had not been shown. MCM1 (also called SRF) plays an important role in the pathogenesis of human diseases and contributes to the metastasis and colonization of $\mathrm{BC}$ cells. It has been reported that the suppressor of cancer cell invasion (SCAI) protein can form a complex with MRTF and SRF to inhibit the invasion of human $\mathrm{BC}$ cells [36]. In addition, knocking out MRTFA subtypes or MCM1 reduces the targeted migration and invasion of human BC (MDA-MB-231) cells [37], indicating that MCM1 plays key roles in the distant metastasis of BC. In our research, we found that low expression of MCM1 in $\mathrm{BC}$ was closely associated with good prognosis for $\mathrm{BC}$ patients.

High MCM2 expression is related to $\mathrm{BC}$ with a high histological grade, while low expression level of MCM2 increases the possibility of RFS in patients with BC [38]. Some reports suggest that MCM2 and MCM3 may be used as substitutes for Ki-67 to measure the proliferation of BC cells and predict prognosis $[39,40]$. Our study showed that MCM2 was upregulated in $\mathrm{BC}$ and its upregulation was significantly associated with poor prognosis for $\mathrm{BC}$ patients. 


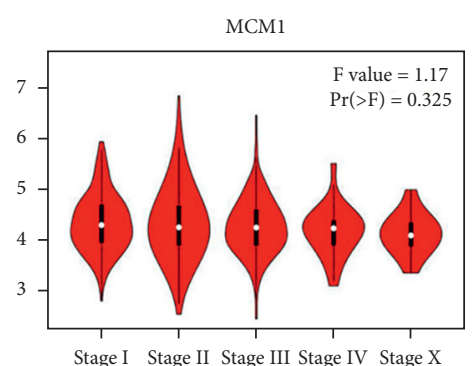

Stage I Stage II Stage III Stage IV Stage X

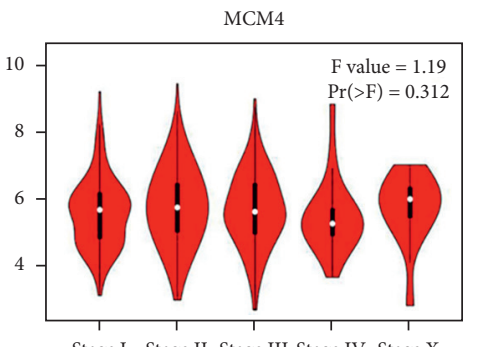

Stage I Stage II Stage III Stage IV Stage X

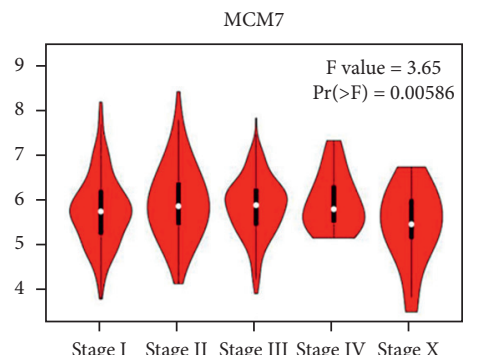

MCM10

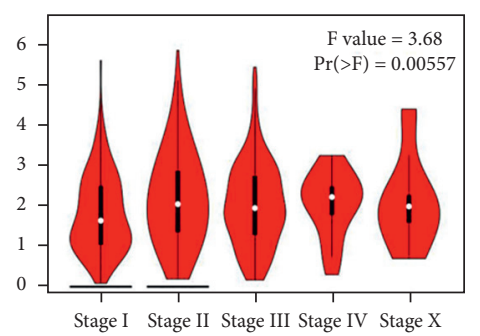

MCM2

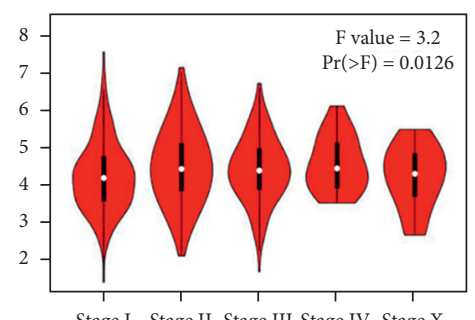

MCM5

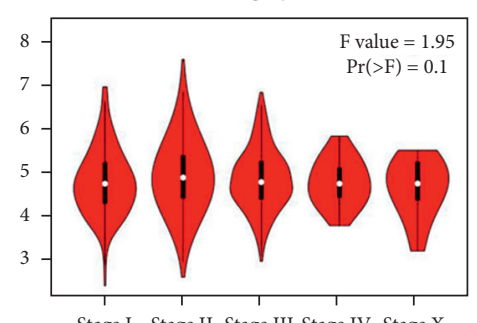

MCM8

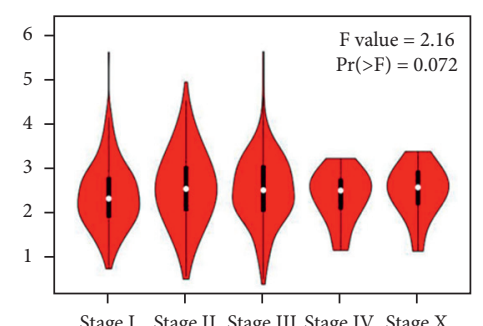

Stage I Stage II Stage III Stage IV Stage X

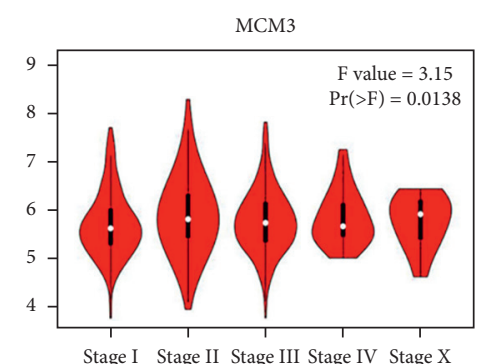

MCM6

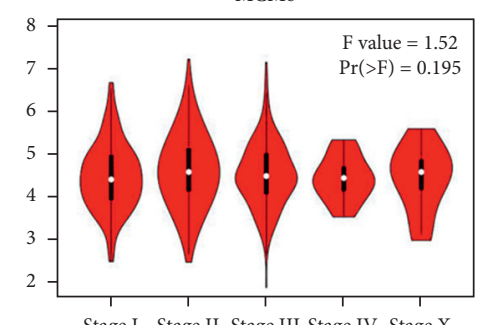

MCM9

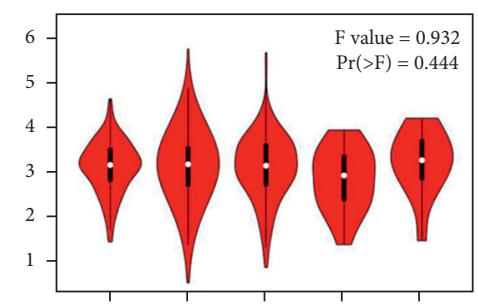

Stage I Stage II Stage III Stage IV Stage X

FIGURE 3: Correlation between MCM expression and tumor stage in breast cancer patients.

Some studies reported that MCM3 was significantly upregulated in $\mathrm{BC}$ and recommended it as a substitute for $\mathrm{Ki}-67$ to measure the proliferation of $\mathrm{BC}$ cells and predict prognosis $[41,42]$. In our study, we also proved that the MCM3 was overexpressed in BC. However, we did not find a significant relationship between high MCM3 expression and the prognosis of BC patients, which still needs further validation with clinical data.

It has been reported that the MCM4 expression level in clinical samples of BC is significantly higher than that in non-tumor breast epithelium $[38,42]$. Shima et al. isolated a subtype mutation of MCM4 called Chaos3 (3 chromosomal aberrations occurring spontaneously). Chaos3 mutations destabilize the MCM2-7 complexes, leading to impaired DNA replication, which increases the risk of $\mathrm{BC}$ in the population [43]. We found that MCM4 was overexpressed in BC. In addition, high MCM4 expression was significantly related to shorter RFS in BC patients, suggesting the prognostic value of MCM4 in BC patients. The study of Issac et al. [38] also supports our view.

While the expression of MCM5 has been accurately reported in other cancers, it has not been done so in $\mathrm{BC}$. For example, the high MCM5 expression is associated with the malignant state and a poor prognosis in cervical adenocarcinoma patients and regulates the proliferation of cervical adenocarcinoma cells [44]. Elevated levels of MCM5 in urine sediment can be used to strongly predict bladder cancer [45]. Our research revealed that the MCM5 expression level in BC was upregulated. In addition, high MCM5 expression was significantly related to low RFS in BC patients, indicating the prognostic value of MCM5 in BC patients.

A high mRNA expression level of MCM6 is related to BC with a high histological grade [38]. In our research, we found 


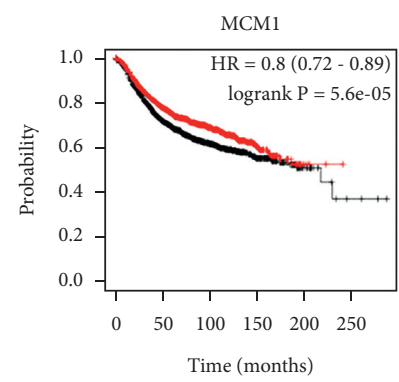

Number at risk

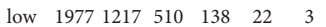

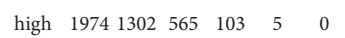

Expression

- low

— high

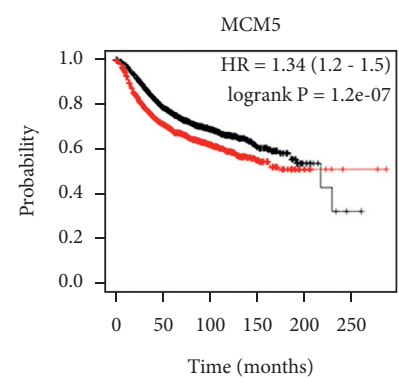

Number at risk

low $19761323 \quad 560 \quad 133 \quad 14 \quad 1$

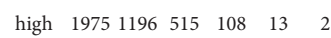

Expression

low

- high

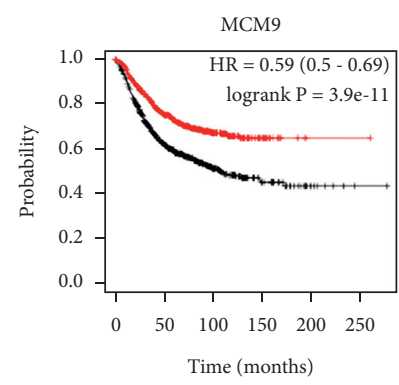

Number at risk

low $\quad 894 \quad 453 \quad 152 \quad 46 \quad 9 \quad 1$

$\begin{array}{lllllll}\text { high } & 870 & 524 & 193 & 22 & 1 & 1\end{array}$

Expression

low

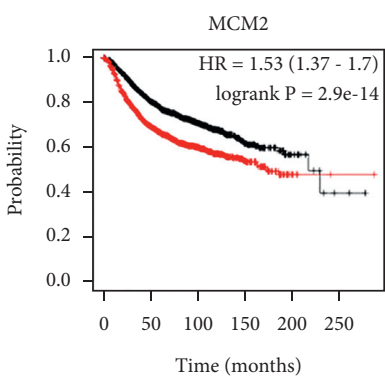

Number at risk

low $\quad \begin{array}{llllll}1976 & 1384 & 641 & 134 & 18 & 2\end{array}$ high $\quad \begin{array}{llllll}1975 & 1135 & 434 & 107 & 9 & 1\end{array}$

Expression

- low

— high

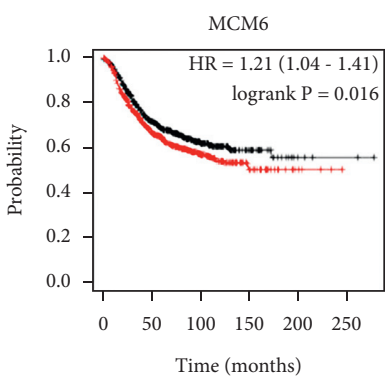

Number at risk

$\begin{array}{lllllll}\text { low } & 885 & 509 & 170 & 37 & 4 & 2\end{array}$

$\begin{array}{lllllll}\text { high } & 879 & 468 & 175 & 31 & 6 & 0\end{array}$

Expression

- low

— high

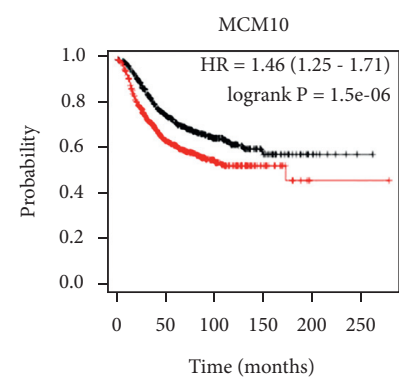

Number at risk

$\begin{array}{lllllll}\text { low } & 885 & 553 & 212 & 46 & 9 & 1\end{array}$

$\begin{array}{lllllll}\text { high } & 879 & 424 & 133 & 22 & 1 & 1\end{array}$

Expression

low

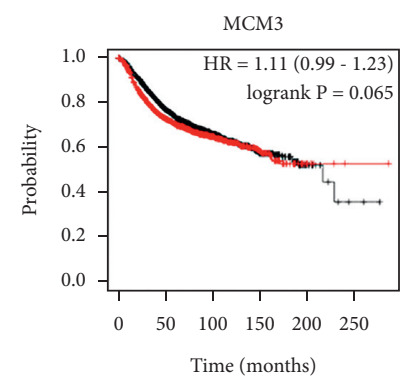

Number at risk

low $\begin{array}{llllll}1977 & 1311 & 533 & 129 & 17 & 2\end{array}$

high $\quad \begin{array}{llllll}1974 & 1208 & 542 & 112 & 10 & 1\end{array}$

Expression

- low

- high

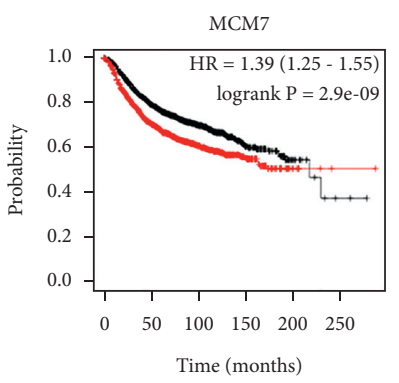

Number at risk

low $\begin{array}{llllll}1981 & 1361 & 611 & 128 & 17 & 2\end{array}$

high $\quad \begin{array}{llllll}1970 & 1158 & 464 & 113 & 10 & 1\end{array}$

Expression

- low

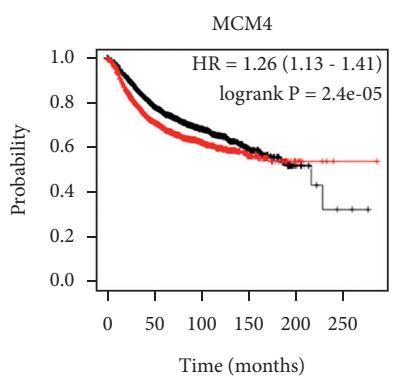

Number at risk

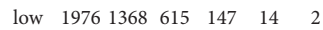

high $\begin{array}{llllll}1975 & 1151 & 460 & 94 & 13 & 2\end{array}$

Expression

- low

— high

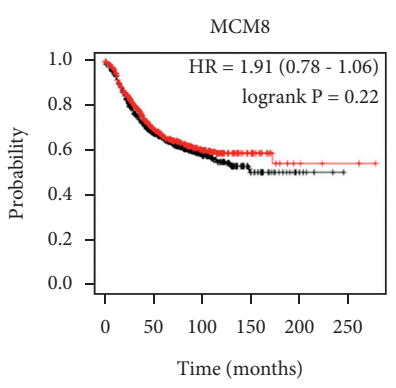

Number at risk

$\begin{array}{lllllll}\text { low } & 883 & 509 & 171 & 35 & 5 & 0\end{array}$

$\begin{array}{lllllll}\text { high } & 881 & 468 & 174 & 33 & 5 & 2\end{array}$

Expression

low

FIgURE 4: Prognostic value of the expression levels of MCMs in patients with BC (Kaplan-Meier plotter).

that MCM6 was significantly increased in BC. In addition, high mRNA expression of MCM6 was significantly related to shorter RFS for BC patients, indicating the prognostic value of MCM6 expression in BC patients. The study of Issac et al. [38] also supports our view.

Huang et al. showed that epidermal growth factor receptor enhanced MCM7-mediated DNA replication through the tyrosine phosphorylation of Lyn kinase in human cancers [46]. Li et al. reported that trifluoropyridine significantly downregulates the expression of PCNA, MCM7, and antiapoptotic Bcl2 in TNBC cells and selectively inhibits the growth of TNBC [47]. Our research found that the expression of MCM7 in BC was upregulated and significantly related to shorter RFS in BC patients, revealing the prognostic value of MCM7 in BC patients.

MCM8 and MCM9 are paralogs of the MCM2-7 replication helicases [48] and are related to $\mathrm{HR}$ in mitotic 


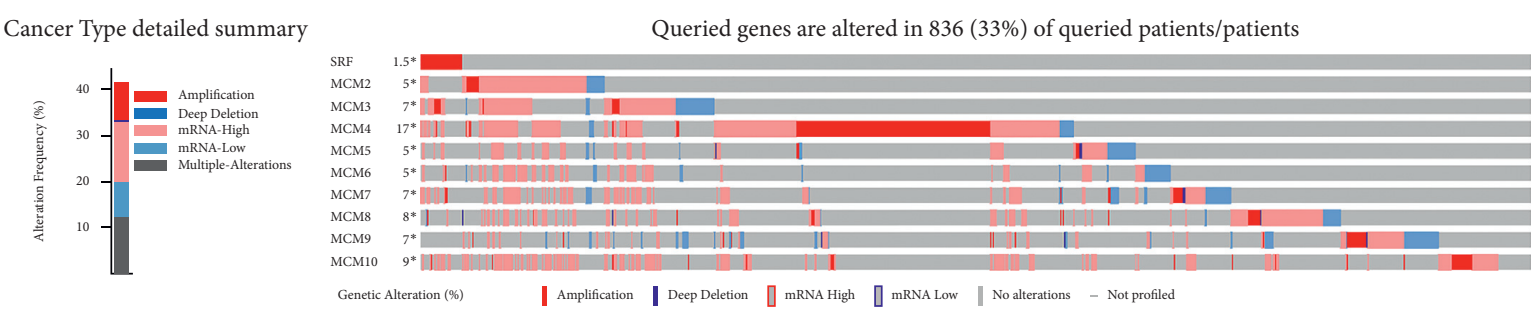

(a)

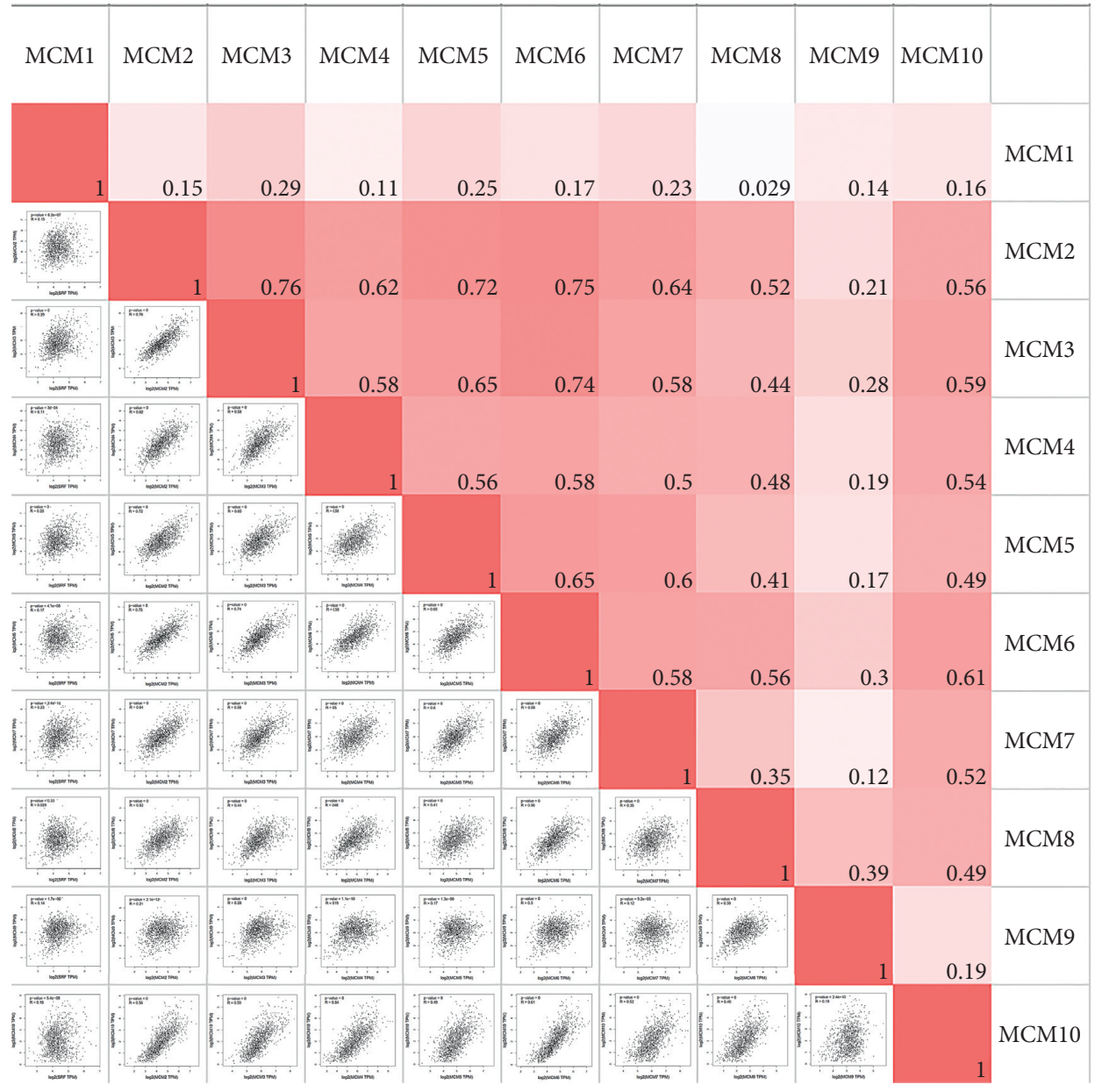

(b)

FIGURE 5: Analysis of alterations and correlation between members of MCMs in BC. (a) Gene expression and alteration analysis of MCMs in BC (cBioPortal). (b) Correlation analysis between different MCMs in BC (GEPIA).

and meiotic cells [49-51]. In addition, MCM8 and MCM9 can cause hematopoietic DNA damage, leading to p53dependent medullary tumors [52]. However, there is no relevant report in BC. Our research showed that the MCM8 expression level in BC was significantly upregulated. However, no significant correlation between high MCM8 expression and RFS in BC patients was found. In contrast, MCM9 was not upregulated in BC and we found that high MCM9 expression was significantly related to longer RFS in BC patients, indicating that MCM9 has good prognostic value in $\mathrm{BC}$ patients.

Currently, it is known that the cell cycle regulation interaction between MCM10 and the dihexamers MCM2-7 is necessary for helicase division and $\mathrm{S}$ phase activation [53]. Yang et al. proved that MCM10 promoted the invasion/ migration potential of $\mathrm{BC}$ cells through $\mathrm{Wnt} / \beta$-catenin signaling and was positively associated with the poor prognosis of BC [54]. In our study, we found that MCM10 was overexpressed in $\mathrm{BC}$ and significantly associated with shorter RFS in BC patients, indicating that MCM10 has good prognostic value in $\mathrm{BC}$ patients.

Studies have shown that abnormally expressed microRNAs can become a sign of cancer. MicroRNA expression is significantly related to tumor occurrence, progression, and treatment, indicating that they may become prognostic and predictive markers [55]. We identified 13 


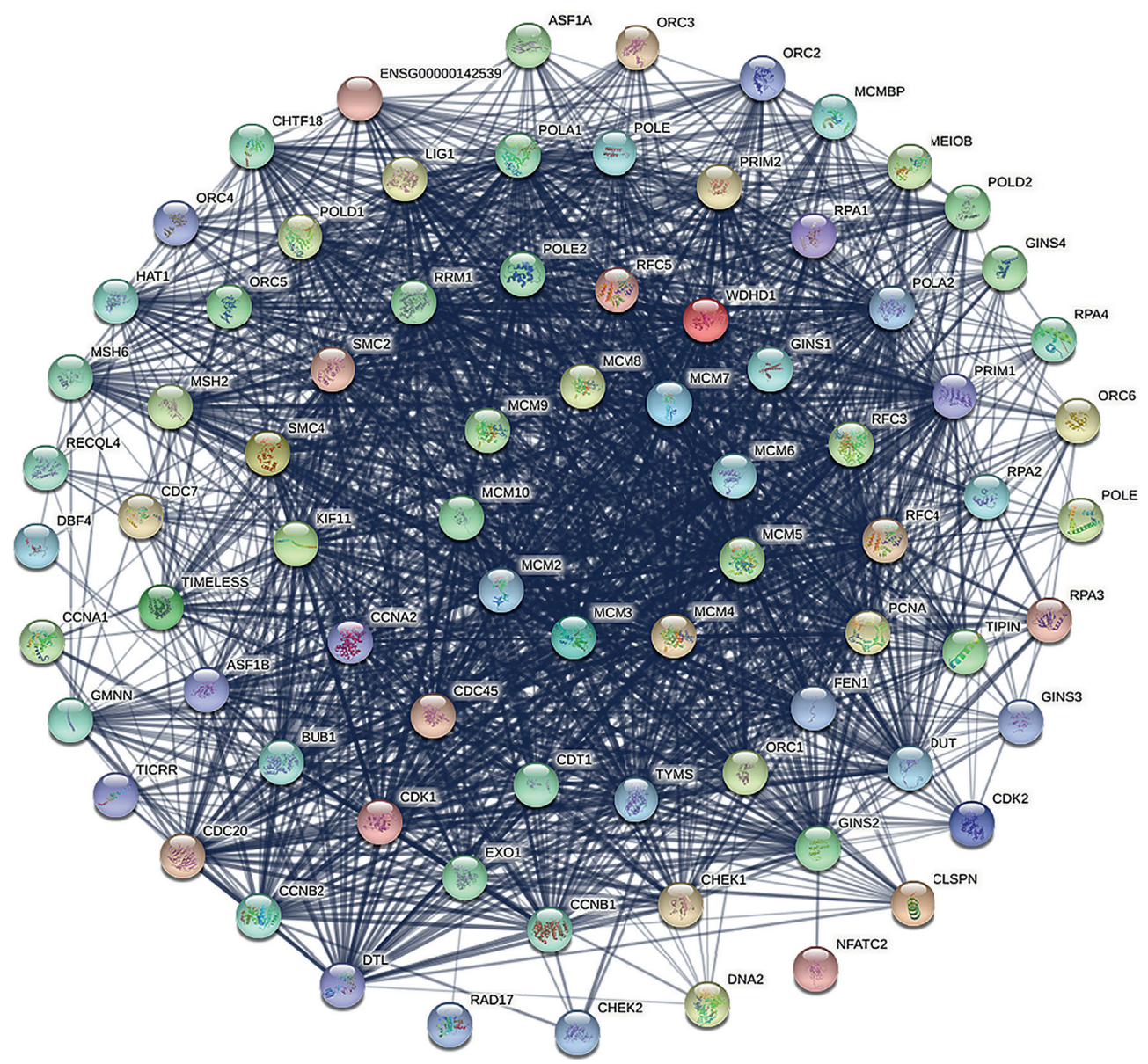

(a)

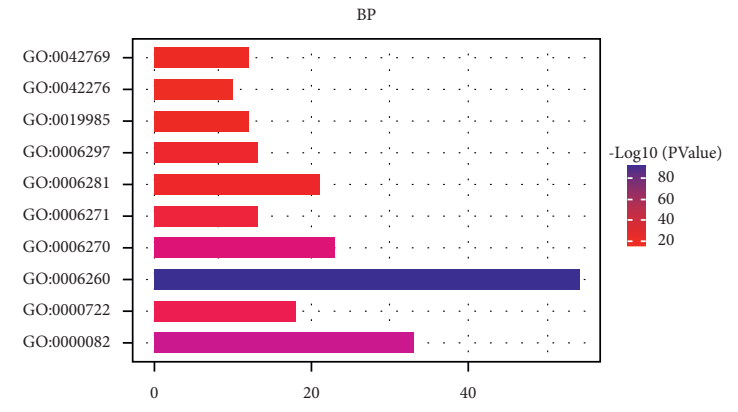

(b)

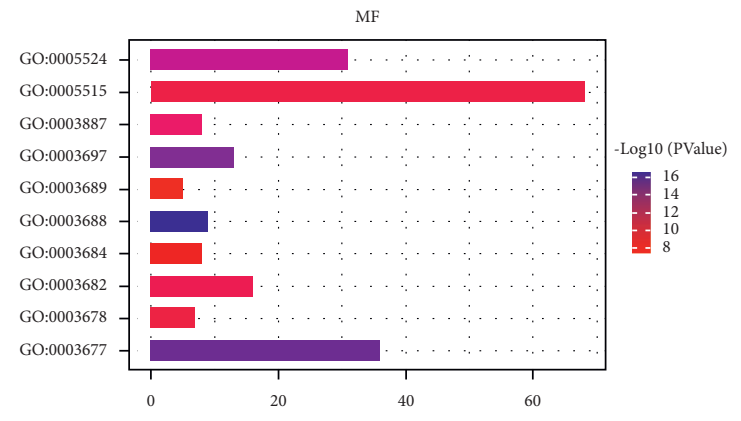

(d)

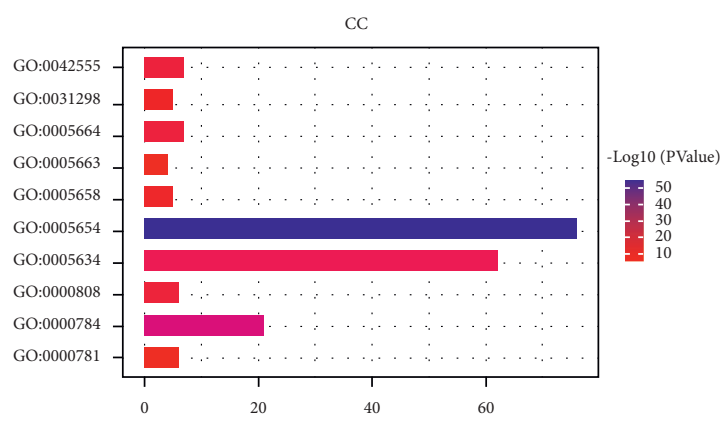

(c)

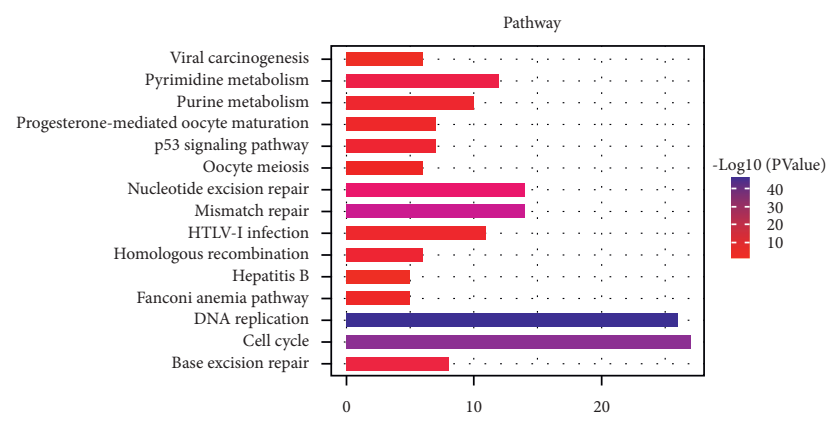

(e)

FIGURE 6: Protein-protein interaction network and functional annotation analysis of MCMs in BC. (a) The protein-protein interaction network of MCMs and frequently altered coexpressed genes (STRING). The potential functions of MCMs and coexpressed genes significantly related to MCMs were predicted based on DAVID and included (b) BP, (c) CC, (d) MF, and (e) KEGG pathways. 


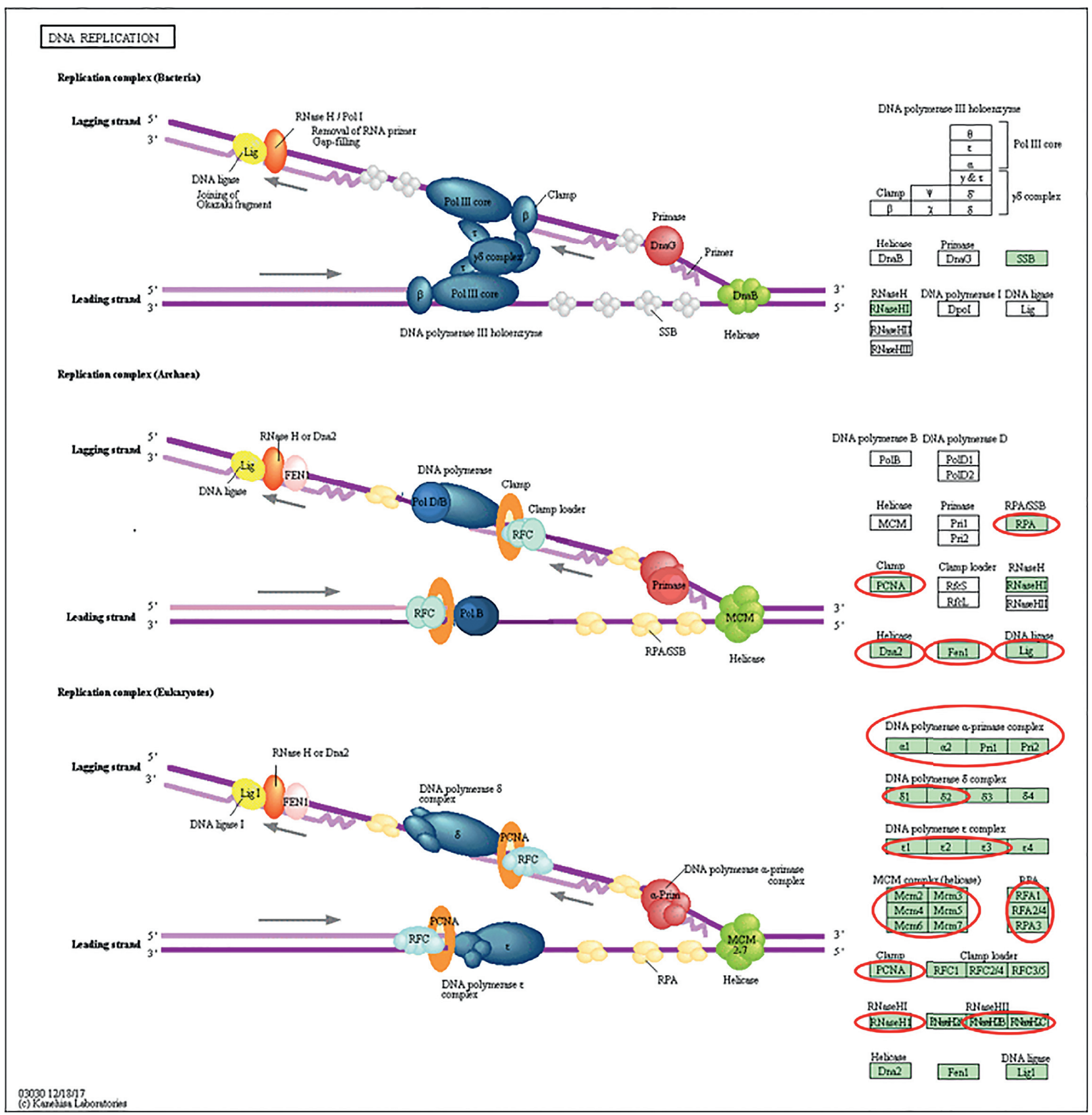

Figure 7: Visualization genes on DNA replication map.

negatively correlated miRNA-MCM pairs, including hsamiR-760-MCM1, hsa-miR-1224-5p-MCM1, hsa-miR-4739MCM1, hsa-miR-139-5p-MCM2, hsa-miR-299-3p-MCM4, hsa-miR-654-5p-MCM4, hsa-miR-140-3p-MCM4, hsamiR-139-5p-MCM5, hsa-miR-326-MCM5, hsa-miR-6545p-MCM5, hsa-miR-299-3p-MCM10, hsa-miR-485-3pMCM10, and hsa-miR-543-MCM10, that may regulate $\mathrm{MCM}$ expression in $\mathrm{BC}$ and be used to predict the prognosis of BC patients.

Functionally, miRNA-760 inhibits the proliferation and metastasis of BC cells by downregulating NANOG and mediates chemoresistance by inhibiting the epithelialmesenchymal transition of $\mathrm{BC}$ cells. Lv et al. reported that
MCF-7 human BC cells overexpressing miR-760 are resistant to Adriamycin [56-58]. In addition, hsa-miR-1224-5p can be used as a valuable treatment target for glioblastoma multiforme [59]. However, the function of hsa-miR-4739 in $\mathrm{BC}$ has not been reported. The loss of Opa-interacting protein 5 can inhibit BC proliferation by the miR-139-5p/ NOTCH1 pathway [60].

Furthermore, hsa-miR-299-3p may play a key role in thyroid cancer [61]. Hsa-miR-654-5p regulates the osteogenic differentiation of human bone marrow mesenchymal stem cells by inhibiting bone morphogenetic protein 2 [62]. The increased expression of hsa-miR-140-3p 5'isomiR contributes to tumor suppressor effect of hsa-miR-140-3p by 


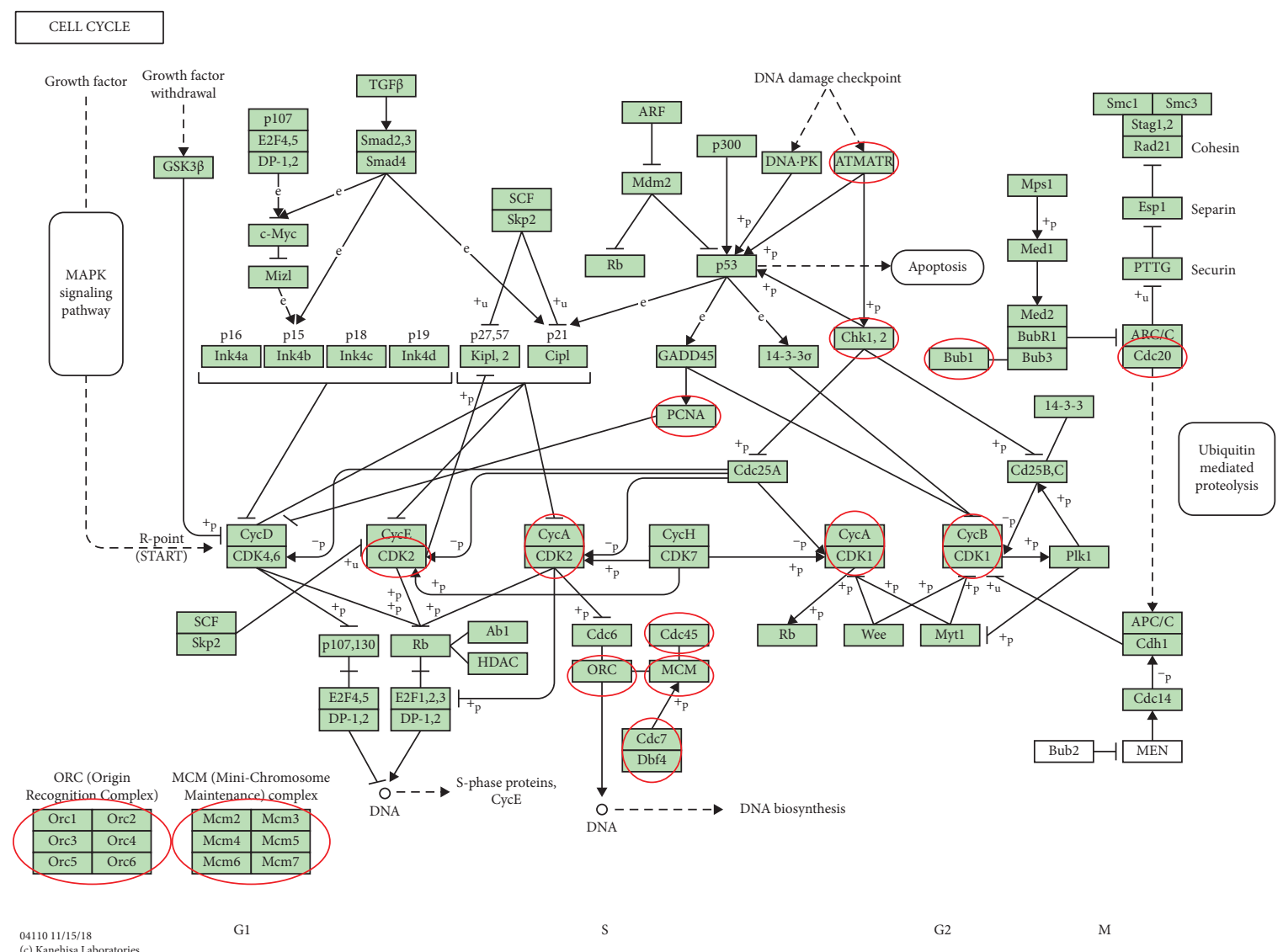

Figure 8: Visualization genes on cell cycle map.

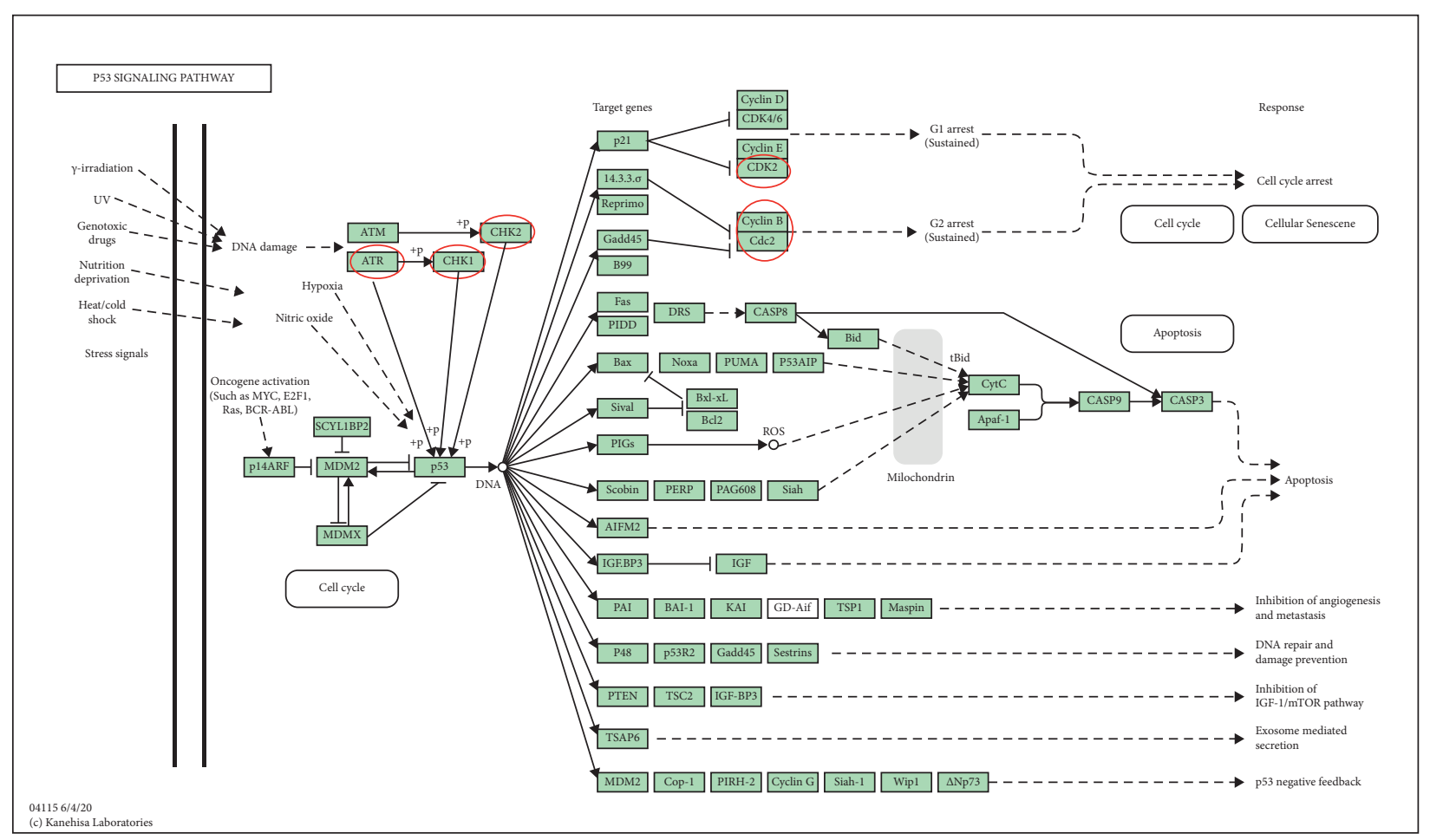

Figure 9: Visualization genes on p53 pathway map. 

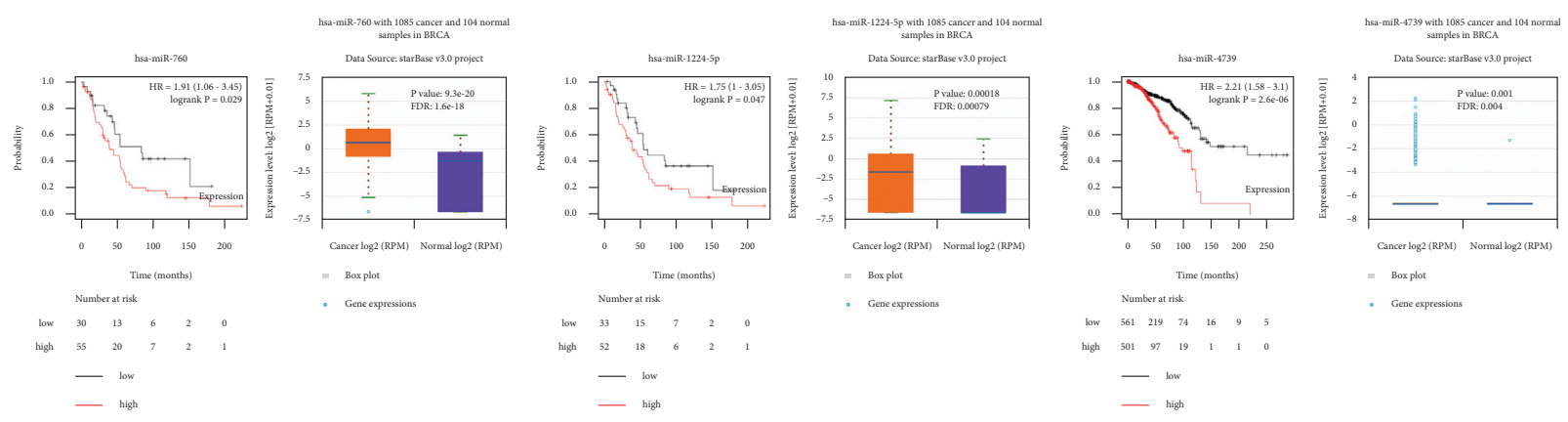

(a)

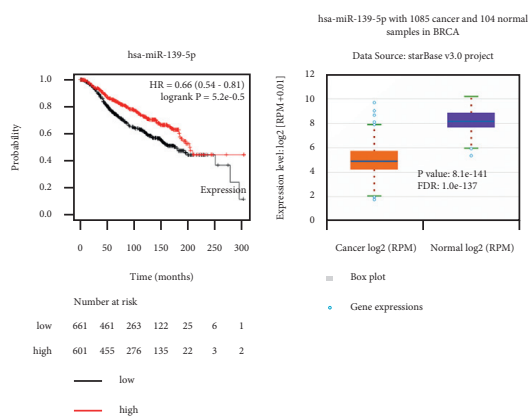

(b)
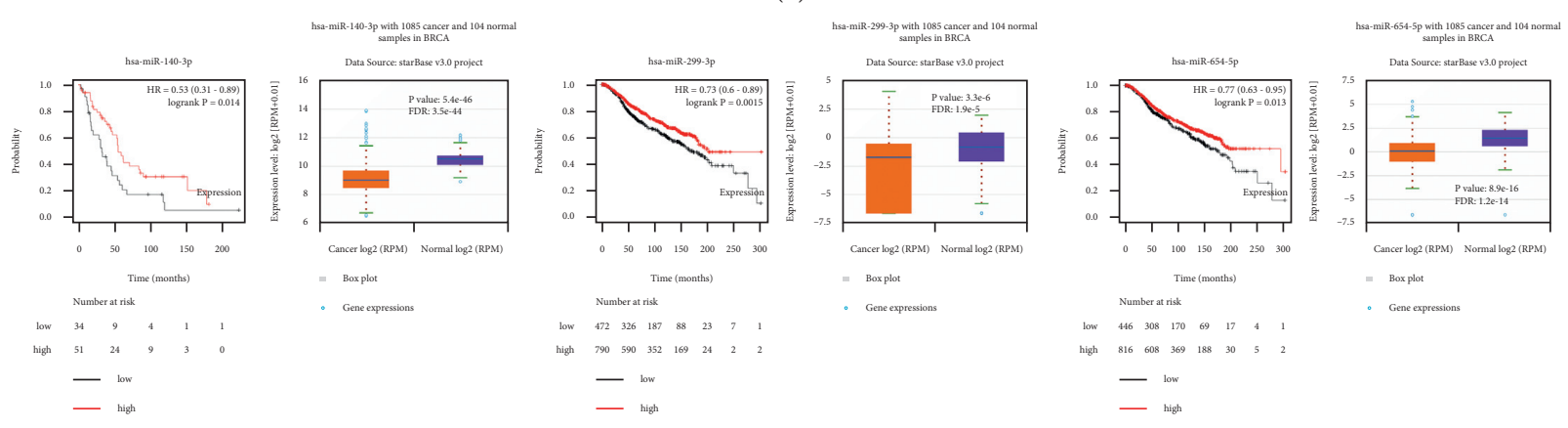

(c)
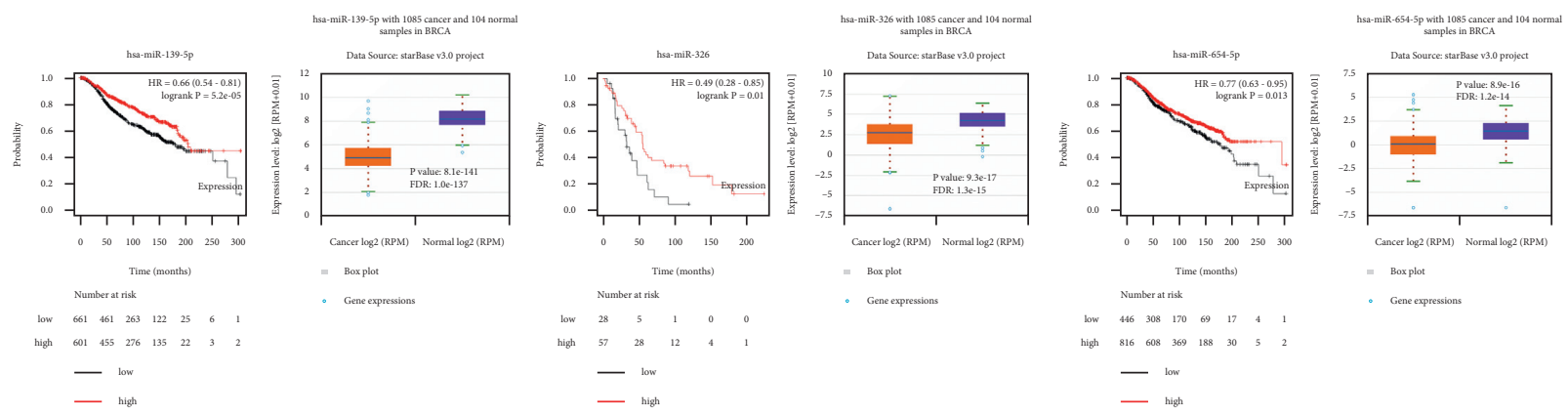

(d)

Figure 10: Continued. 

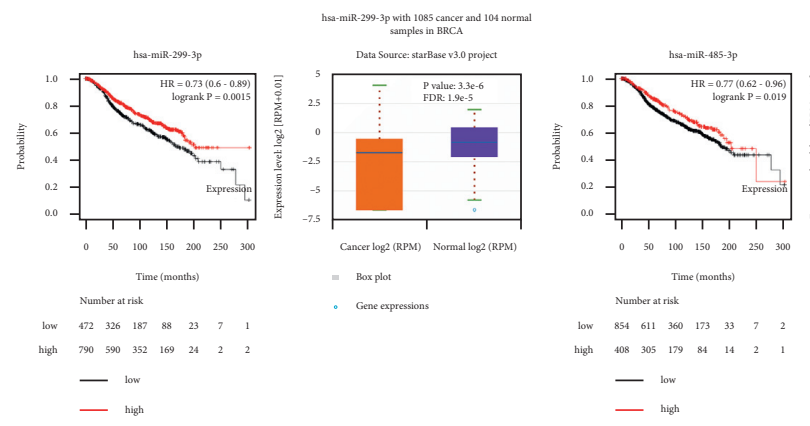

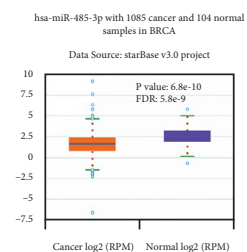

Box plot

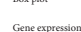

-

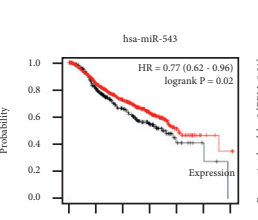

$\begin{array}{llllll}50 & 100 & 150 & 200 & 250 & 300\end{array}$

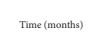

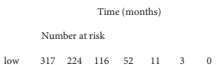

$\begin{array}{lllllll}\text { high } & 945 & 692 & 423 & 205 & 36 & 6\end{array}$

- low

(e)

Figure 10: The miRNAs that associated with prognosis in BC patients. (a) has-miR-760, has-miR-1224-5p, and has-miR-4739 predicted poor overall survival in BC patients. (b) has-miR-139-5p predicted good overall survival in BC patients. (c) has-miR-1403p, has-miR-299-3p, and has-miR-654-5p predicted good overall survival in BC patients. (d) has-miR-139-5p, has-miR-326, and hasmiR-654-5p predicted good overall survival in BC patients. (e) has-miR-229-3p, has-miR-485-3p, and has-miR-543 predicted good overall survival in BC patients.

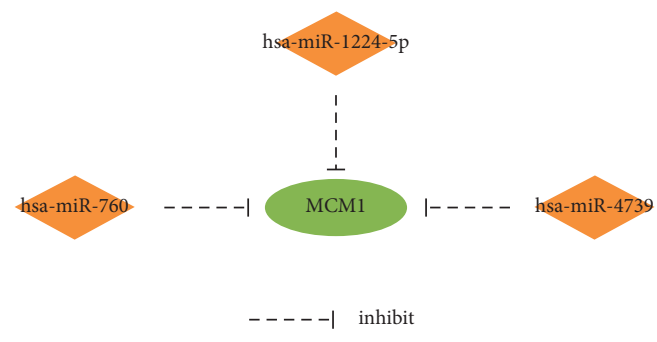

(a)

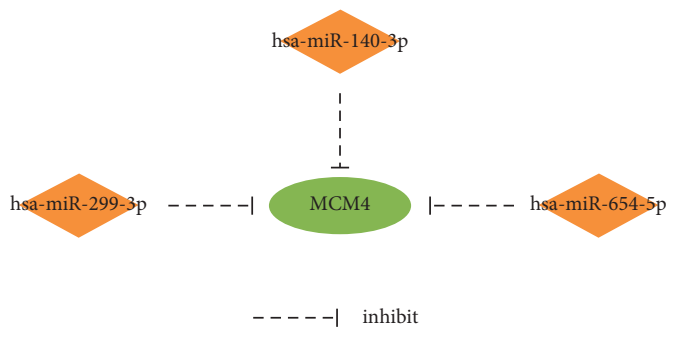

(c)

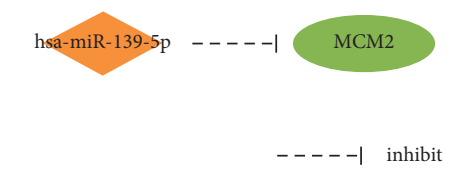

(b)

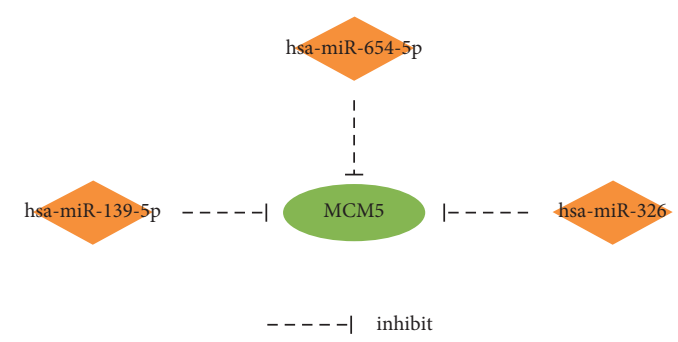

(d)

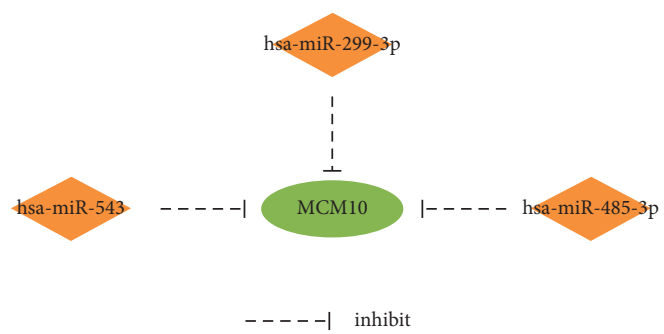

(e)

FIGURE 11: miRNA-mRNA regulation network. (a) miRNA-MCM1 regulation network. (b) miRNA-MCM2 regulation network. (c) miRNA-MCM4 regulation network. (d) miRNA-MCM5 regulation network. (e) miRNA-MCM10 regulation network.

reducing the proliferation and migration of $\mathrm{BC}$ [63]. HsamiR-326 participates in the chemotherapy resistance of $\mathrm{BC}$ by regulating the expression of multidrug resistance-related protein 1 [64]. Hsa-miR-326 can inhibit BC by targeting SOX12, making miR-326 a promising therapeutic target for BC [65]. Hsa-miR-543 functions as a carcinoma suppressor in glioma [66]. Moreover, Yu et al. revealed that hsa-miR543 functions as a tumor suppressor in ovarian carcinoma by targeting TWIST1 [67]. In addition, hsa-miR-543 can target TRPM7 to inhibit cervical cancer [68].

Our findings suggest that MCM family members play crucial roles in BC progression, and MCM2, MCM4-7, and 
MCM10 in tumor tissues possess great potential to be valuable prognostic biomarkers for BC patients. Clinically, the detection of these protein biomarkers mainly depends on tissue biopsy, which is an invasive method and not always feasible or repeatable. With the development of liquid biopsy, these limitations are being gradually overcome. Liquid biopsy is a rapid, comprehensive, and non-invasive detection method and allows for the longitudinal assessment of cancer evolution. A blood-based liquid biopsy has been reported to efficiently capture circulating tumor cells (CTCs) and circulating tumor-derived nucleic acids, including circulating tumor DNA (ctDNA) [69-72]. CTCs are cancer cells originating from the primary tumor and metastatic sites, which are found in the blood. Increasing evidence shows that CTCs act as valuable biomarkers with high sensitivity and specificity to monitor therapeutic efficacy and predict prognosis in metastatic BC $[73,74]$. Due to the crucial role of MCM family members in DNA homeostasis, MCMs are probably involved in the regulation of CTC biological behavior. Therefore, investigating the expression pattern and function of MCMs in different phenotype of CTCs may provide new insight into evaluating the therapeutic efficacy and prognosis of BC patients. ctDNA is released from cancer cells and contains tumor-specific genetic and epigenetic alterations. It has been reported that the mutations or alterations of genes in ctDNA are closely associated with curative effect of therapies in $\mathrm{BC}[74,75]$. ctDNA analysis may provide an excellent tool to monitor therapeutic efficacy and predict prognosis. For instance, the analysis of ESR1 mutations in ctDNA of metastatic BC patients can be used to predict resistance to endocrine therapy [76]. The detection of PIK3CA alterations in plasmaderived ctDNA and PIK3CA ctDNA levels predicts the response of $\mathrm{BC}$ patients to palbociclib and fulvestrant therapy [77]. Moreover, analysis of HER2 mutation frequency in ctDNA can be used to predict response of $\mathrm{BC}$ patients to neratinib with high sensitivity and specificity [78]. In addition, ctDNA fraction and somatic copy-number alterations are correlated with significantly worse outcomes in triple-negative BC patients $[69,79]$. These studies provide great help for clinicians to adjust the appropriate therapeutic strategy for BC patients in time. Our data from functional annotation analysis revealed that MCMs are involved in the regulation of several pathways, including nucleotide excision repair, mismatch repair, DNA replication, and base excision repair. This means that dysregulation of MCMs might be involved in the occurrence of mutation or alteration of genes in DNA. Future studies are required to investigate the detailed mechanisms of MCMs involved in the occurrence of gene mutation or alteration, which may facilitate the clinical application of ctDNA and development of new therapeutic strategies for BC patients.

\section{Conclusion}

In this study, we systematically analyzed the expression levels, clinicopathological characteristics, functions, and prognostic values of MCMs in BC. Our results suggest that upregulated expression levels of MCM2-8 and MCM10 in
BC samples play important roles in BC. High expression of MCM2, MCM4-7, and MCM10 shows great potential to be molecular markers to identify patients with BC. Additionally, MCM1 and MCM9 also exhibit the possibility as prognostic markers for improving the survival rate of $\mathrm{BC}$ patients and prognostic accuracy. The miRNAs regulating MCM1, MCM2, MCM4-7, and MCM10 can be involved in carcinogenesis and improve the prognosis of $\mathrm{BC}$ patients. However, there are still some limitations in our study. For instance, only Kaplan-Meier plotter was used to evaluate the prognostic value of MCMs in BC. A multivariable Cox model is required to further validate these findings in future study.

\section{Data Availability}

The data used to support the findings of this study can be obtained through the corresponding author upon reasonable request.

\section{Conflicts of Interest}

The authors declare that they have no conflicts of interest.

\section{Authors' Contributions}

Xinyu Liu and Ying Liu contributed equally to this study.

\section{Acknowledgments}

We thank the National Natural Science Foundation of China (31670989), Key R\&D Program of Shandong Province (2019GSF107037), and Science and Technology Project of Qingdao, China (18-6-1-88-nsh), for their support.

\section{Supplementary Materials}

Table S1: correlation of miRNA-MCM1 pairs identified by ENCORI database. Table S2: correlation of miRNA-MCM2 pairs identified by ENCORI database. Table S3: correlation of miRNA-MCM4 pairs identified by ENCORI database. Table S4: correlation of miRNA-MCM5 pairs identified by ENCORI database. Table S5: correlation of miRNA-MCM6 pairs identified by ENCORI database. Table S6: correlation of miRNA-MCM7 pairs identified by ENCORI database. Table S7: correlation of miRNA-MCM9 pairs identified by ENCORI database. Table S8: correlation of miRNA-MCM10 pairs identified by ENCORI database. (Supplementary Materials)

\section{References}

[1] R. L. Siegel, K. D. Miller, and A. Jemal, "Cancer statistics, 2019,” CA: A Cancer Journal for Clinicians, vol. 69, no. 1, pp. 7-34, 2019.

[2] R. L. Siegel, K. D. Miller, and A. Jemal, "Cancer statistics, 2020,” CA: A Cancer Journal for Clinicians, vol. 70, no. 1, pp. 7-30, 2020.

[3] T. Sorlie, C. M. Perou, R. Tibshirani et al., "Gene expression patterns of breast carcinomas distinguish tumor subclasses 
with clinical implications," Proceedings of the National Academy of Sciences, vol. 98, no. 19, pp. 10869-10874, 2001.

[4] S. R. D. Johnston, "Clinical efforts to combine endocrine agents with targeted therapies against epidermal growth factor receptor/human epidermal growth factor receptor 2 and mammalian target of rapamycin in breast cancer," Clinical Cancer Research, vol. 12, no. 3, pp. 1061s-1068s, 2006.

[5] E. R. Jenkinson and J. P. J. Chong, "Minichromosome maintenance helicase activity is controlled by $\mathrm{N}$ - and C-terminal motifs and requires the ATPase domain helix-2 insert," Proceedings of the National Academy of Sciences, vol. 103, no. 20, pp. 7613-7618, 2006.

[6] S. E. Kearsey and K. Labib, "MCM proteins: evolution, properties, and role in DNA replication," Biochimica et Biophysica Acta (BBA)-Gene Structure and Expression, vol. 1398, no. 2, pp. 113-136, 1998.

[7] D. I. Lin, P. Aggarwal, and J. A. Diehl, "Phosphorylation of MCM3 on Ser-112 regulates its incorporation into the MCM2-7 complex," Proceedings of the National Academy of Sciences, vol. 105, no. 23, pp. 8079-8084, 2008.

[8] S. Vijayraghavan and A. Schwacha, "The eukaryotic Mcm2-7 replicative helicase," Subcellular Biochemistry, vol. 62, pp. 113-134, 2012.

[9] S. Chattopadhyay and A.-K. Bielinsky, "Human Mcm10 regulates the catalytic subunit of DNA polymerase- $\alpha$ and prevents DNA damage during replication," Molecular Biology of the Cell, vol. 18, no. 10, pp. 4085-4095, 2007.

[10] Y. Chen, C. Weng, H. Zhang, J. Sun, and Y. Yuan, "A direct interaction between P53-binding protein 1 and minichromosome maintenance complex in Hepg2 cells," Cellular Physiology and Biochemistry, vol. 47, no. 6, pp. 2350-2359, 2018.

[11] J. Gao, Q. Wang, C. Dong, S. Chen, Y. Qi, and Y. Liu, "Whole exome sequencing identified MCM2 as a novel causative gene for autosomal dominant nonsyndromic deafness in a Chinese family," PLoS One, vol. 10, no. 7, Article ID e0133522, 2015.

[12] W. Wu, X. Wang, C. Shan, Y. Li, and F. Li, "Minichromosome maintenance protein 2 correlates with the malignant status and regulates proliferation and cell cycle in lung squamous cell carcinoma," OncoTargets and Therapy, vol. 11, pp. 5025-5034, 2018.

[13] H. Zhong, B. Chen, H. Neves et al., "Expression of minichromosome maintenance genes in renal cell carcinoma," Cancer Management and Research, vol. 9, pp. 637-647, 2017.

[14] P. A. Stewart, Z. I. Khamis, H. E. Zhau et al., "Upregulation of minichromosome maintenance complex component 3 during epithelial-to-mesenchymal transition in human prostate cancer," Oncotarget, vol. 8, no. 24, pp. 39209-39217, 2017.

[15] A. Wojnar, B. Pula, A. Piotrowska et al., "Correlation of intensity of MT-I/II expression with Ki-67 and MCM-2 proteins in invasive ductal breast carcinoma," Anticancer Research, vol. 31, pp. 3027-3033, 2011.

[16] C. Giaginis, M. Georgiadou, K. Dimakopoulou et al., "Clinical significance of MCM-2 and MCM-5 expression in colon cancer: association with clinicopathological parameters and tumor proliferative capacity," Digestive Diseases and Sciences, vol. 54, no. 2, pp. 282-291, 2009.

[17] Y.-P. Peng, Y. Zhu, L.-D. Yin et al., "The expression and prognostic roles of MCMs in pancreatic cancer," PLoS One, vol. 11, no. 10, Article ID e0164150, 2016.

[18] K. Shomori, K. Nishihara, T. Tamura et al., "Geminin, Ki67, and minichromosome maintenance 2 in gastric hyperplastic polyps, adenomas, and intestinal-type carcinomas: pathobiological significance," Gastric Cancer, vol. 13, no. 3, pp. 177-185, 2010.

[19] H.-Q. Cai, Z.-J. Cheng, H.-P. Zhang et al., "Overexpression of MCM6 predicts poor survival in patients with glioma," $\mathrm{Hu}$ man Pathology, vol. 78, pp. 182-187, 2018.

[20] A. Marnerides, T. P. Vassilakopoulos, E. Boltetsou et al., "Immunohistochemical expression and prognostic significance of CCND3, MCM2 and MCM7 in Hodgkin lymhoma," Anticancer Research, vol. 31, pp. 3585-3594, 2011.

[21] D. R. Rhodes, J. Yu, K. Shanker et al., "ONCOMINE: a cancer microarray database and integrated data-mining platform," Neoplasia, vol. 6, no. 1, pp. 1-6, 2004.

[22] D. R. Rhodes, S. Kalyana-Sundaram, V. Mahavisno et al., "Oncomine 3.0: genes, pathways, and networks in a collection of 18,000 cancer gene expression profiles," Neoplasia, vol. 9, no. 2, pp. 166-180, 2007.

[23] Z. Tang, C. Li, B. Kang, G. Gao, C. Li, and Z. Zhang, "GEPIA: a web server for cancer and normal gene expression profiling and interactive analyses," Nucleic Acids Research, vol. 45, no. W1, pp. W98-w102, 2017.

[24] B. Györffy, P. Surowiak, J. Budczies, and A. Lánczky, “Online survival analysis software to assess the prognostic value of biomarkers using transcriptomic data in non-small-cell lung cancer," PLoS One, vol. 8, Article ID e82241, 2013.

[25] E. Cerami, J. Gao, U. Dogrusoz et al., "The cBio cancer genomics portal: an open platform for exploring multidimensional cancer genomics data: figure 1," Cancer Discovery, vol. 2, no. 5, pp. 401-404, 2012.

[26] C. V. Mering, M. Huynen, D. Jaeggi, S. Schmidt, P. Bork, and B. Snel, "STRING: a database of predicted functional associations between proteins," Nucleic Acids Research, vol. 31, no. 1, pp. 258-261, 2003.

[27] G. Dennis Jr., B. T. Sherman, D. A. Hosack et al., "DAVID: database for annotation, visualization, and integrated discovery," Genome Biology, vol. 4, no. 5, p. P3, 2003.

[28] J.-H. Li, S. Liu, H. Zhou, L.-H. Qu, and J.-H. Yang, "starBase v2.0: decoding miRNA-ceRNA, miRNA-ncRNA and proteinRNA interaction networks from large-scale CLIP-Seq data," Nucleic Acids Research, vol. 42, no. D1, pp. D92-D97, 2014.

[29] C. Curtis, S. P. Shah, S. P. Shah et al., "The genomic and transcriptomic architecture of 2,000 breast tumours reveals novel subgroups," Nature, vol. 486, no. 7403, pp. 346-352, 2012.

[30] T. Sorlie, R. Tibshirani, J. Parker et al., "Repeated observation of breast tumor subtypes in independent gene expression data sets," Proceedings of the National Academy of Sciences of the United States of America, vol. 100, pp. 8418-8423, 2003.

[31] C. M. Perou, T. Sørlie, M. B. Eisen et al., "Molecular portraits of human breast tumours," Nature, vol. 406, no. 6797, pp. 747-752, 2000.

[32] S. Glück, J. S. Ross, M. Royce et al., “TP53 genomics predict higher clinical and pathologic tumor response in operable early-stage breast cancer treated with docetaxel-capecitabine \pm trastuzumab," Breast Cancer Research and Treatment, vol. 132, no. 3, pp. 781-791, 2012.

[33] A. L. Richardson, Z. C. Wang, A. De Nicolo et al., "X chromosomal abnormalities in basal-like human breast cancer," Cancer Cell, vol. 9, no. 2, pp. 121-132, 2006.

[34] L. Radvanyi, D. Singh-Sandhu, S. Gallichan et al., "The gene associated with trichorhinophalangeal syndrome in humans is overexpressed in breast cancer," Proceedings of the National Academy of Sciences, vol. 102, no. 31, pp. 11005-11010, 2005.

[35] G. Turashvili, J. Bouchal, K. Baumforth et al., "Novel markers for differentiation of lobular and ductal invasive breast 
carcinomas by laser microdissection and microarray analysis," BMC Cancer, vol. 7, no. 1, p. 55, 2007.

[36] D. T. Brandt, C. Baarlink, T. M. Kitzing et al., "SCAI acts as a suppressor of cancer cell invasion through the transcriptional control of $\beta 1$-integrin," Nature Cell Biology, vol. 11, no. 5, pp. 557-568, 2009.

[37] S. Medjkane, C. Perez-Sanchez, C. Gaggioli, E. Sahai, and R. Treisman, "Myocardin-related transcription factors and SRF are required for cytoskeletal dynamics and experimental metastasis," Nature Cell Biology, vol. 11, no. 3, pp. 257-268, 2009.

[38] M. S. M. Issac, E. Yousef, M. R. Tahir, and L. A. Gaboury, "MCM2, MCM4, and MCM6 in breast cancer: clinical utility in diagnosis and prognosis," Neoplasia, vol. 21, no. 10, pp. 1015-1035, 2019.

[39] E. M. Yousef, D. Furrer, D. L. Laperriere et al., "MCM2: an alternative to Ki-67 for measuring breast cancer cell proliferation," Modern Pathology, vol. 30, no. 5, pp. 682-697, 2017.

[40] Y. Zhao, Y. Wang, F. Zhu, J. Zhang, X. Ma, and D. Zhang, "Gene expression profiling revealed MCM3 to be a better marker than Ki67 in prognosis of invasive ductal breast carcinoma patients," Clinical and Experimental Medicine, vol. 20, no. 2, pp. 249-259, 2020.

[41] S.-A. Ha, S. M. Shin, H. Namkoong et al., "Cancer-associated expression of Minichromosome maintenance 3Gene in several human cancers and its involvement in tumorigenesis," Clinical Cancer Research, vol. 10, no. 24, pp. 8386-8395, 2004.

[42] H. F. Kwok, S. D. Zhang, C. M. McCrudden et al., "Prognostic significance of minichromosome maintenance proteins in breast cancer," American journal of cancer research, vol. 5, pp. 52-71, 2015.

[43] N. Shima, A. Alcaraz, I. Liachko et al., "A viable allele of $\mathrm{Mcm} 4$ causes chromosome instability and mammary adenocarcinomas in mice," Nature Genetics, vol. 39, no. 1, pp. 93-98, 2007.

[44] D. Wang, Q. Li, Y. Li, and H. Wang, "The role of MCM5 expression in cervical cancer: correlation with progression and prognosis," Biomedicine \& Pharmacotherapy, vol. 98, pp. 165-172, 2018.

[45] K. Stoeber, R. Swinn, A. T. Prevost et al., "Diagnosis of genitourinary tract cancer by detection of minichromosome maintenance 5 protein in urine sediments," Cancer Spectrum Knowledge Environment, vol. 94, no. 14, pp. 1071-1079, 2002.

[46] T.-H. Huang, L. Huo, Y.-N. Wang et al., "Epidermal growth factor receptor potentiates MCM7-mediated DNA replication through tyrosine phosphorylation of Lyn kinase in human cancers," Cancer Cell, vol. 23, no. 6, pp. 796-810, 2013.

[47] J. Li, J. Liu, R. Wang et al., "Trifluridine selectively inhibits cell growth and induces cell apoptosis of triple-negative breast cancer," American journal of cancer research, vol. 10, pp. 507-522, 2020.

[48] Y. Liu, T. A. Richards, and S. J. Aves, "Ancient diversification of eukaryotic MCM DNA replication proteins,” BMC Evolutionary Biology, vol. 9, no. 1, p. 60, 2009.

[49] J. Park, D. T. Long, K. Y. Lee et al., "The MCM8-MCM9 complex promotes RAD51 recruitment at DNA damage sites to facilitate homologous recombination," Molecular and Cellular Biology, vol. 33, no. 8, pp. 1632-1644, 2013.

[50] K. Nishimura, M. Ishiai, K. Horikawa et al., "Mcm8 and $\mathrm{Mcm} 9$ form a complex that functions in homologous recombination repair induced by DNA interstrand crosslinks," Molecular Cell, vol. 47, no. 4, pp. 511-522, 2012.

[51] M. Lutzmann, C. Grey, S. Traver et al., "MCM8- and MCM9deficient mice reveal gametogenesis defects and genome instability due to impaired homologous recombination," Molecular Cell, vol. 47, no. 4, pp. 523-534, 2012.

[52] M. Lutzmann, F. Bernex, C. da Costa de Jesus et al., "MCM8and MCM9 deficiencies cause lifelong increased hematopoietic DNA damage driving p53-dependent myeloid tumors," Cell Reports, vol. 28, no. 11, pp. 2851-2865, 2019.

[53] Y. Quan, Y. Xia, L. Liu et al., "Cell-cycle-regulated interaction between $\mathrm{Mcm} 10$ and double hexameric $\mathrm{mcm} 2-7$ is required for helicase splitting and activation during $\mathrm{S}$ phase," Cell Reports, vol. 13, no. 11, pp. 2576-2586, 2015.

[54] W. D. Yang and L. Wang, "MCM10 facilitates the invaded/ migrated potentials of breast cancer cells via $\mathrm{Wnt} / \beta$-catenin signaling and is positively interlinked with poor prognosis in breast carcinoma," Journal of Biochemical and Molecular Toxicology, vol. 33, Article ID e22330, 2019.

[55] M. V. Iorio and C. M. Croce, "MicroRNA dysregulation in cancer: diagnostics, monitoring and therapeutics: a comprehensive review," EMBO Molecular Medicine, vol. 4, no. 3, pp. 143-159, 2012.

[56] S. H. Hu, C. H. Wang, Z. J. Huang et al., "miR-760 mediates chemoresistance through inhibition of epithelial mesenchymal transition in breast cancer cells," European Review for Medical and Pharmacological Sciences, vol. 20, pp. 5002-5008, 2016.

[57] J. Lv, Z. Fu, M. Shi et al., "Systematic analysis of gene expression pattern in has-miR-760 overexpressed resistance of the MCF-7 human breast cancer cell to doxorubicin," Biomedicine \& Pharmacotherapy, vol. 69, pp. 162-169, 2015.

[58] M.-L. Han, F. Wang, Y.-T. Gu et al., "MicroR-760 suppresses cancer stem cell subpopulation and breast cancer cell proliferation and metastasis: by down-regulating NANOG," Biomedicine \& Pharmacotherapy, vol. 80, pp. 304-310, 2016.

[59] D. D. Xiong, W. Q. Xu, R. Q. He, Y. W. Dang, G. Chen, and D. Z. Luo, "In silico analysis identified miRNA-based therapeutic agents against glioblastoma multiforme," Oncology Reports, vol. 41, pp. 2194-2208, 2019.

[60] H.-C. Li, Y.-F. Chen, W. Feng et al., "Loss of the Opa interacting protein 5 inhibits breast cancer proliferation through miR-139-5p/NOTCH1 pathway," Gene, vol. 603, pp. 1-8, 2017.

[61] X. Liu, M. He, Y. Hou et al., "Expression profiles of microRNAs and their target genes in papillary thyroid carcinoma," Oncology Reports, vol. 29, no. 4, pp. 1415-1420, 2013.

[62] J. Q. Wei, H. Chen, X. F. Zheng et al., "Hsa-miR-654-5p regulates osteogenic differentiation of human bone marrow mesenchymal stem cells by repressing bone morphogenetic protein 2," Nan Fang Yi Ke Da Xue Xue Bao, vol. 32, pp. 291-295, 2012.

[63] O. Salem, N. Erdem, J. Jung et al., "The highly expressed 5 'isomiR of hsa-miR-140-3p contributes to the tumor-suppressive effects of miR-140 by reducing breast cancer proliferation and migration," BMC Genomics, vol. 17, no. 1, p. 566, 2016.

[64] Z. Liang, H. Wu, J. Xia et al., "Involvement of miR-326 in chemotherapy resistance of breast cancer through modulating expression of multidrug resistance-associated protein 1," Biochemical Pharmacology, vol. 79, no. 6, pp. 817-824, 2010.

[65] Y. Du, L. Shen, W. Zhang et al., "Functional analyses of microRNA-326 in breast cancer development," Bioscience Reports, vol. 39, Article ID BSR20190787, 2019.

[66] L. Xu, J. Yu, Z. Wang, Q. Zhu, W. Wang, and Q. Lan, “miR543 functions as a tumor suppressor in glioma in vitro and in vivo," Oncology Reports, vol. 38, no. 2, pp. 725-734, 2017. 
[67] Q. Yu, Z. Zhang, B. He, H. Wang, P. Shi, and Y. Li, "MiR-543 functions as tumor suppressor in ovarian cancer by targeting TWIST1," Journal of Biological Regulators and Homeostatic Agents, vol. 34, pp. 101-110, 2020.

[68] X. Liu, L. Gan, and J. Zhang, "miR-543 inhibites cervical cancer growth and metastasis by targeting TRPM7," ChemicoBiological Interactions, vol. 302, pp. 83-92, 2019.

[69] M. Russano, A. Napolitano, G. Ribelli et al., "Liquid biopsy and tumor heterogeneity in metastatic solid tumors: the potentiality of blood samples," Journal of Experimental o Clinical Cancer Research, vol. 39, no. 1, p. 95, 2020.

[70] T. Tayoun, V. Faugeroux, M. Oulhen, A. Aberlenc, P. Pawlikowska, and F. Farace, "CTC-derived models: a window into the seeding capacity of circulating tumor cells (CTCs)," Cells, vol. 8, no. 10, p. 1145, 2019.

[71] L. Keller, Y. Belloum, H. Wikman, and K. Pantel, "Clinical relevance of blood-based ctDNA analysis: mutation detection and beyond," British Journal of Cancer, vol. 124, no. 2, pp. 345-358, 2021.

[72] Y. Liu, "Targeting the non-canonical AKT-FOXO3a axis: a potential therapeutic strategy for oral squamous cell carcinoma," EBioMedicine, vol. 49, pp. 6-8, 2019.

[73] D. H. Liang, C. Hall, and A. Lucci, "Circulating tumor cells in breast cancer," Tumor Liquid Biopsies, vol. 215, pp. 127-145, 2020.

[74] S. Alimirzaie, M. Bagherzadeh, and M. R. Akbari, "Liquid biopsy in breast cancer: a comprehensive review," Clinical Genetics, vol. 95, no. 6, pp. 643-660, 2019.

[75] F. Clatot, "Review ctDNA and breast cancer," Tumor Liquid Biopsies, vol. 215, pp. 231-252, 2020.

[76] C. Fribbens, B. O’Leary, L. Kilburn et al., "Plasma ESR1 mutations and the treatment of estrogen receptor-positive advanced breast cancer," Journal of Clinical Oncology, vol. 34, no. 25, pp. 2961-2968, 2016.

[77] B. O’Leary, S. Hrebien, J. P. Morden et al., "Early circulating tumor DNA dynamics and clonal selection with palbociclib and fulvestrant for breast cancer," Nature Communications, vol. 9, p. 896, 2018.

[78] C. X. Ma, R. Bose, F. Gao et al., "Neratinib efficacy and circulating tumor DNA detection of HER2 mutations in HER2 nonamplified metastatic breast cancer," Clinical Cancer Research, vol. 23, no. 19, pp. 5687-5695, 2017.

[79] D. G. Stover, H. A. Parsons, G. Ha et al., "Association of cellfree DNA tumor fraction and somatic copy number alterations with survival in metastatic triple-negative breast cancer," Journal of Clinical Oncology, vol. 36, no. 6, pp. 543-553, 2018. 